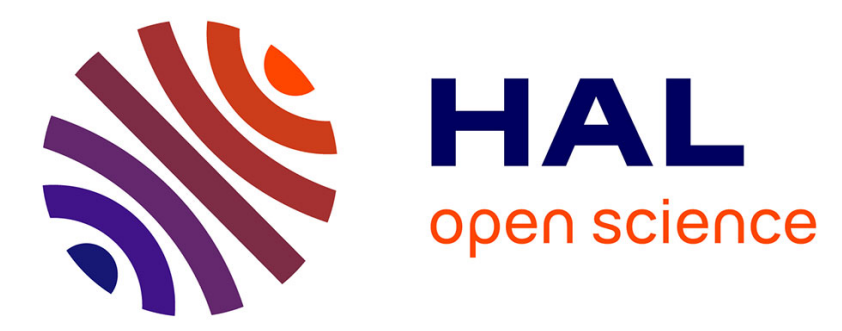

\title{
Vision-based adaptive assistance and haptic guidance for safe wheelchair corridor following
}

\author{
Vishnu Karakkat Narayanan, François Pasteau, Maud Marchal, Alexandre
} Krupa, Marie Babel

\section{- To cite this version:}

Vishnu Karakkat Narayanan, François Pasteau, Maud Marchal, Alexandre Krupa, Marie Babel. Vision-based adaptive assistance and haptic guidance for safe wheelchair corridor following. Computer Vision and Image Understanding, 2016, 179, pp.171-185. 10.1016/j.cviu.2016.02.008 . hal-01277585

\section{HAL Id: hal-01277585 \\ https://hal.inria.fr/hal-01277585}

Submitted on 22 Feb 2016

HAL is a multi-disciplinary open access archive for the deposit and dissemination of scientific research documents, whether they are published or not. The documents may come from teaching and research institutions in France or abroad, or from public or private research centers.
L'archive ouverte pluridisciplinaire HAL, est destinée au dépôt et à la diffusion de documents scientifiques de niveau recherche, publiés ou non, émanant des établissements d'enseignement et de recherche français ou étrangers, des laboratoires publics ou privés. 


\title{
Vision-based adaptive assistance and haptic guidance for safe wheelchair corridor following
}

\author{
Vishnu K.Narayanan ${ }^{\mathrm{a}}$, François Pasteau ${ }^{\mathrm{b}}$, Maud Marchal ${ }^{\mathrm{c}}$, Alexandre \\ Krupa $^{\mathrm{a}}$, Marie Babel ${ }^{\mathrm{c}}$ \\ ${ }^{a}$ Inria Rennes - Bretagne Atlantique and IRISA, France. \\ ${ }^{b}$ Ergovie, France. \\ ${ }^{c}$ Inria Rennes - Bretagne Atlantique, INSA Rennes and IRISA, France.
}

\begin{abstract}
In case of motor impairments, steering a wheelchair can become a hazardous task. Joystick jerks induced by uncontrolled motions may lead to wall collisions when a user steers a wheelchair along a corridor. This work introduces a low-cost assistive and guidance system for indoor corridor navigation in a wheelchair, which uses purely visual information, and which is capable of providing automatic trajectory correction and haptic guidance in order to avoid wall collisions. A visual servoing approach to autonomous corridor following serves as the backbone to this system. The algorithm employs natural image features which can be robustly extracted in real time. This algorithm is then fused with manual joystick input from the user so that progressive assistance and trajectory correction can be activated as soon as the user is in danger of collision. A force feedback in conjunction with the assistance is provided on the joystick in order to guide the user out of his dangerous trajectory. This ensures intuitive guidance and minimal interference from the trajectory correction system. In addition to being a low-cost approach, it can be seen that the proposed solution does not require an a-priori environment model. Experiments on a robotised wheelchair equipped with a monocular camera prove the capability of the system to adaptively guide and assist a user navigating in a corridor.
\end{abstract}

Keywords: Vision-based robotics, Visual servoing, Assistive robotics, Wheelchair navigation

Email address: vishnu.karakkat-narayanan@inria.fr (Vishnu K.Narayanan) 


\section{Introduction}

Wheelchairs are versatile assistive devices that are able to address virtually all physically disabling pathologies, and they help millions of people in achieving mobility. But severe motor disabilities like Parkinson's disease as well as visual impairments prevent people from steering a wheelchair effectively, particularly in constrained environments [1]. In addition, difficulties appear during long-term driving. Depending on the handicap, steering a wheelchair along a corridor can then become a difficult task especially when corridors are narrow enough to induce an uncomfortable sensation in navigation $[2]$.

It is well known that individual mobility is an integral part of the mental and social well-being of a disabled person [3]. Therefore smart devices have to be designed in order to help a wheelchair user navigate efficiently without the help of another person. One can say that recent advances in robotics have facilitated the enhancement of a simple electric wheelchair into a smart wheelchair. Here the idea is to assist a user in fundamental navigation tasks like corridor following, doorway passing and to perform local obstacle avoidance. Different projects like the TAO Project [4], the NavChair [5], European FP7 Radhar project [6] and the recent SYSIASS project [2] were indeed able to design systems that take partial/full control from the user for safe and effective navigation assistance.

The aim of this paper is to then introduce an adaptive assistive and guidance system in order to prevent wall collisions when a user manually steers a wheelchair in a corridor. While the systems mentioned above consider a multi-sensor architecture and known maps of the operating environment, the proposed solution relies on low-cost architecture which includes a single monocular camera and a haptic joystick. Moreover, the general direction taken in Smart Wheelchair design is to propose a complete system built from the ground up. While some recent works introduce adaptable designs for increasing the assistive capabilities in an off-the-shelf electric wheelchair $[4$, $5,6,7,8]$, the systems are tested and validated on specially built wheelchairs or mobile robots with the exception of a few projects which adapt existing powered wheelchairs with the requisite software or hardware $[2,9,10]$.

Therefore the aim here is to design a modular system tested on an off-theshelf electric wheelchair. In addition, a fully vision-based setup along with 
a map-less design facilitates easier commercialization and widespread usage. Thus the goal is to design a robust vision-based control system that could be used in order to augment user teleoperation to derive a semi-autonomous solution for corridor following. Such semi-autonomous or shared control wheelchair systems would be helpful in conditions where motor impairments may hinder secure and effective navigation, by providing safe assistance in performing fundamental tasks like corridor following while maintaining high level control with the user.

A variety of solutions have been proposed in the area of corridor following with mobile/wheeled robots [11]. While specifically considering vision-based corridor following using a mobile robot, in [12] a visual approach was designed using an omni-directional camera with the system utilizing a visual memory framework. Furthermore, in [13] two vision-based control algorithms for corridor navigation were presented that exploited the geometry of a typical corridor. The first one used the optical flow measured from the corridor's lateral walls to generate an angular velocity command for the mobile robot. The second scheme found the perspective lines of the walls meeting the floor to generate the angular velocity command for the robot. Moreover [14] demonstrates a stable image-based and position-based controller for autonomous mobile robot navigation in corridors that considers parallel perspective lines on the floor. Whereas in [15], visual servoing based on a vanishing point detection is fused with appearance-based process to monitor and control the position of a mobile robot in a corridor. Therefore, the geometry of a corridor in an image is a viable solution to employ while designing vision-based algorithms (or visual tasks) around it for realising the task of corridor following.

But this visual task has to be blended with manual control/user intention so that the user maintains a higher level control over the wheelchair motion. Most smart wheelchair designs give the high-level control (e.g. goal selection, path planning) to the user, and the low-level control (e.g. motion control commands, obstacle avoidance) to the robot $[2,4,5,6,16]$. Recently, a variety of solutions have been proposed that use non-invasive Brain-Computer Interfaces (BCI) for assessing the user intent and then augment the user intent using external sensors for safe navigation [9, 10, 17]. Using BCIs may provide an accurate estimate of user intention, but the major drawback lies in the fact that the user must concentrate very hard to convey his intent. This may be a difficult task, especially for people with motor disabilities. On the other hand, using voice [18] and/or gaze [19] as user tele-operation have 
limited scope in terms of modularity as well. Therefore straightforward and modular solution would be to blend manual control from a joystick with robot control in order to create a co-operative/collaborative system with the user as the high level controller. This concept was demonstrated in [16] where the manual control from the joystick was augmented with an autonomous controller capable of obstacle avoidance. Moreover in [7], a linear control blending formalism was introduced in order to fuse user and robot control for assisted doorway traversal in a wheelchair.

Also, to maximize the acceptability of the assistance solution, this assistance has to be progressively activated only when necessary and to be deactivated as soon as the user wants to act by himself [1]. As a consequence, the control process has to be designed as a man-in-the-loop scheme where the user remains the leader of the navigation process and automatic trajectory correction is adaptively activated when in danger.

In conjunction with automatic trajectory correction, a guiding joystick force is necessary in the case where users suffer from visual and/or cognitive impairments and are not able to clearly observe their unsafe trajectory. It can also be seen as a communication channel between the user and the wheelchair controller for a better user experience where such an active feedback can lead to minimal interference from the automatic trajectory correction system [20]. This concept of haptic feedback for wheelchair navigation assistance has been previously explored mainly as a mechanism for obstacle avoidance where the feedback was calculated from the classical potential field method $[21,22,23,24]$. Recently in the context of the European FP7 Radhar project, haptic feedback was provided in order to achieve a bilateral guidance channel where the haptic controller relays the intention of the system so that the user is able to overrule actions if needed [20]. Therefore it is efficient to provide a force feedback which is in conjunction or in proportion with the automatic trajectory correction so that there is an intuitive form of communication with the user.

The proposed work therefore presents an image-based control scheme to integrate an autonomous visual navigation task with user teleoperation while a user is manually driving a wheelchair. This provides progressive assistance whenever the user is in danger of collision. In addition, a guiding force, which is also explicitly modelled from visual information, is applied on the joystick in order to notify the user of his/her unsafe trajectory. The system is then analysed on an off-the-shelf wheelchair equipped with a monocular camera and a haptic joystick. 
Consequently, Section 2 details the proposed approach and contributions of the work. Section 3 presents the geometric modelling of the system, Section 4 illustrates the image features extraction processes and Section 5 explains the image-based controller along with the design of the haptic guidance scheme. The experimental analysis is presented in Section 6.

\section{Proposed System and Contributions}

In this work, we initially design a visual wall collision avoidance task around an Image-Based Visual Servoing scheme that is capable of following corridors autonomously $[25,26,27]$. The visual task employs natural image features including the vanishing point and the vanishing lines that correspond to the wall/floor boundaries, as inputs. We then integrate an assistance solution, that fuses the wall collision avoidance task with user tele-operation output [28]. The fusion formulation is defined in such a way that the task is progressively activated, when the wheelchair gets closer to the walls, in order to steer the wheelchair away from it. This also means that if there

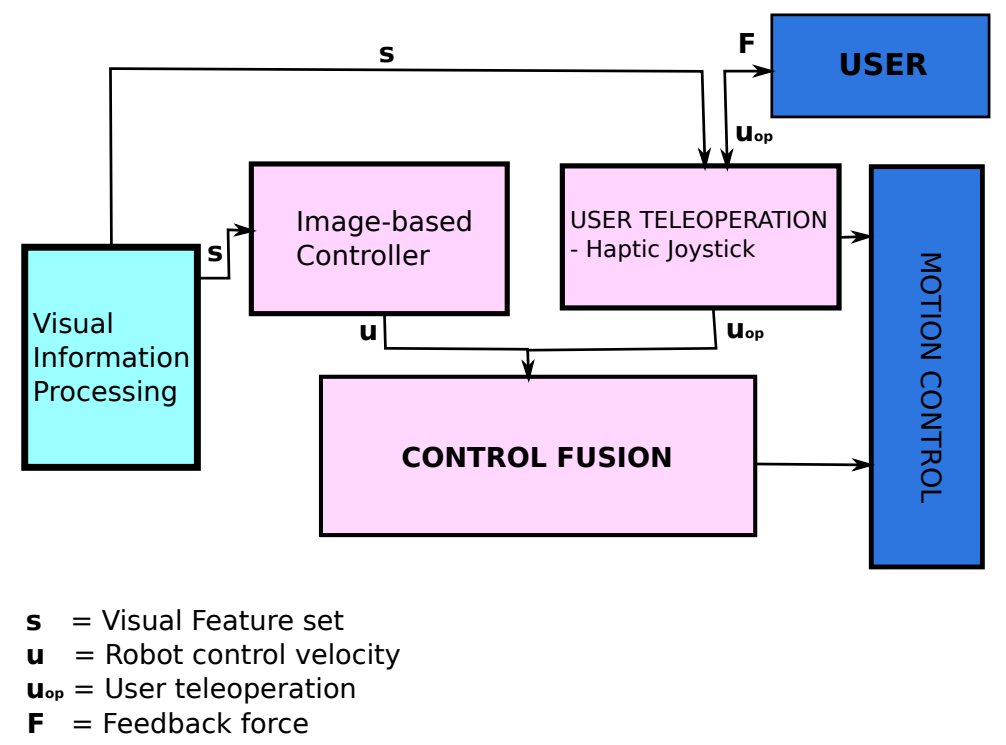

Figure 1: The visual feature set $\mathbf{s}$ derived from a scene is used to drive a visual controller that generates a velocity output $\mathbf{u}$. The robot output is then blended with user teleoperation from the haptic joystick $\mathbf{u}_{o p}$ in order to obtain a collaborative shared control system. Note that the haptic feedback guidance is also modelled from visual information. 
is no threat of collision, the user will have full control over the wheelchair motion. Finally, we design an optimal joystick force feedback in conjunction with the trajectory correction process that helps the user to understand the dangerousness of the situation and which intuitively guides him over to a safe trajectory. The progressive assistance and the force fed back in order to guide the user, are both explicitly modelled from visual information. Therefore the guiding force applied on the joystick will be in conjunction with the automatic correction. This will lead to a more intuitive experience since the feedback force will concurrently help in trajectory correction (thereby decreasing the system effort). An overview of the approach is presented in Figure 1.

As a result, information from an on-board monocular camera is used to explicitly design an assistive semi-autonomous solution for corridor navigation in a wheelchair that also communicates the intention of the provided assistance to the user.

\section{Modelling}

We model the wheelchair as a non-holonomic unicycle type robot that is able to move on a horizontal/inclined plane. Two differential wheels located in the middle of the robot body provide motion while two passive caster wheels each at the front and rear are required for balance. Therefore the two components of the control velocity $(\mathbf{u})$ are the translational component $u$ along its forward/backward direction and the angular (steering) component $\omega$.

From Figure 2, we observe $\mathcal{F}_{r}\left(P_{O}, x_{r}, y_{r}, z_{r}\right)$ as the Cartesian frame of the wheelchair robot with its origin located at the middle of the segment formed by the centers of the two differential wheels, while $\mathcal{F}_{c}\left(C, x_{c}, y_{c}, z_{c}\right)$ states the camera frame (of a front facing calibrated camera) that is rigidly fixed to the wheelchair robot where $C$ represents the optical center of the camera. We define the image frame as $\mathcal{F}_{I}\left(I_{0}, X, Y\right)$ where $I_{0}$ is the center point of the image.

We mount the camera on the wheelchair such that it is at a height $h$ from the floor and that we have a translation vector ${ }^{c} \mathbf{t}_{r}=(w, h,-l)$ between $\mathcal{F}_{r}$ and $\mathcal{F}_{c}$. Since the rotation matrix ${ }^{c} \mathbf{R}_{r}$ that models the fixed orientation of the camera frame relatively to the robot frame is given by

$$
{ }^{c} \mathbf{R}_{r}=\left[\begin{array}{ccc}
0 & -1 & 0 \\
0 & 0 & -1 \\
1 & 0 & 0
\end{array}\right]
$$




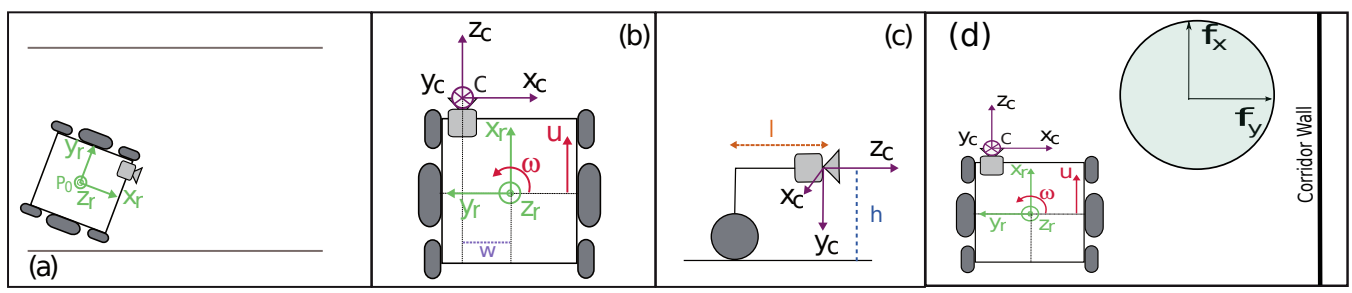

Figure 2: Robot, camera and joystick frames. (a) Top view of the wheelchair with the robot frame. (b) Top view with relative positions of robot and camera frame. (c) Simplified side view. (d) The haptic joystick space showing the direction of the force feedback components with respect to the robot and camera frames

the velocity screw transformation matrix ${ }^{c} \mathbf{W}_{r}$ which links the camera velocity to the robot velocity is given by

$$
{ }^{c} \mathbf{W}_{r}=\left[\begin{array}{cc}
{ }^{c} \mathbf{R}_{r} & {\left[{ }^{c} \mathbf{t}_{r}\right]_{\times}{ }^{c} \mathbf{R}_{r}} \\
0_{3 \times 3} & { }^{c} \mathbf{R}_{r}
\end{array}\right]
$$

with $\left[{ }^{c} \mathbf{t}_{r}\right]_{\times}$representing the skew-symmetric matrix related to the translation vector ${ }^{c} \mathbf{t}_{r}$. The robot Jacobian ${ }^{r} \mathbf{J}_{r}$ expressed in the robot frame is equal to

$$
{ }^{r} \mathbf{J}_{r}=\left[\begin{array}{llllll}
1 & 0 & 0 & 0 & 0 & 0 \\
0 & 0 & 0 & 0 & 0 & 1
\end{array}\right]^{T} .
$$

Finally, we assume a 2-D force frame $\mathcal{F}_{f}\left(F, x_{F}, y_{F}\right)$ for a 2-DOF haptic joystick having the capability of assigning forces in the $x_{F}$ and $y_{F}$ directions as shown in Figure 2d. The feedback force is thus defined as $\mathbf{F}=\left[f_{x}, f_{y}\right]^{T}$.

\section{Visual Features Extraction}

As stated earlier, we design the assistance mechanism over a visual servoing task capable of realising autonomous corridor following [26] [27]. Visual servoing is a well-known framework for robot motion control. The aim of a visual servoing task is to design a robot velocity controller that minimizes the error e between the set of detected visual features $\mathbf{s}$ and a set of their desired values $\mathbf{s}^{*}$ in the image [25]. A generic representation of the relevant natural image features in a corridor is given in Figure 3 from which we could observe some features that could be exploited to design an image based controller. Since the objective is to devise a control law which minimises the error $\mathbf{e}=\left[\mathbf{s}-\mathbf{s}^{*}\right]^{T}$, we have to select features so that the wheelchair is able 
to position the optical axis of camera parallel to the wall and at the middle of the corridor.
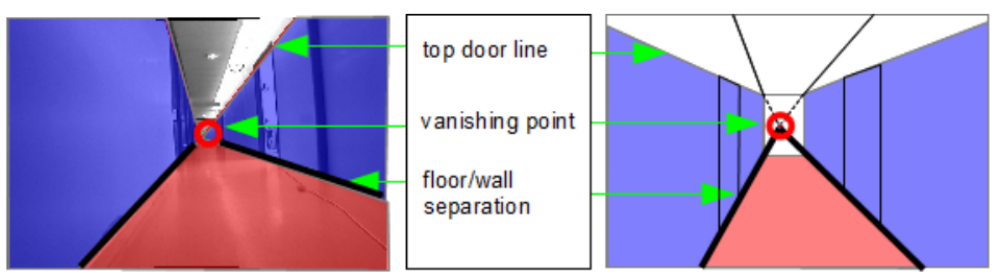

Figure 3: Generic corridor geometrical structure in an image

In [27], we demonstrated a robust and locally asymptotically stable visual servoing scheme that controls the angular velocity $\omega$ of the wheelchair by exponentially minimizing the error e between a set of visual features $\mathbf{s}=\left(x_{f}, \theta_{m}\right)$ and a set of its desired values $\mathbf{s}^{*}=\left(x_{f}^{*}, \theta_{m}^{*}\right)$. This serves as a control system for autonomous corridor following. Here $x_{f}$ represents the $X$-coordinate of the vanishing point $f_{t}=\left(x_{f}, y_{f}\right)$ at an instant $t$ (shown in Figure 4$)$. Whereas $\theta_{m}$ represents the angle made by the $z_{c}$-axis of the camera with the median line of the corridor (see Figure 4). When the camera is positioned parallel to the wall, looking forward, the feature $x_{f}$ reduces to zero and when the position of the camera is in the middle of the corridor, the feature $\theta_{m}$ is equal to zero. Therefore $\mathbf{s}^{*}=\left(x_{f}^{*}, \theta_{m}^{*}\right)=(0,0)$. Consequently if we set a constant translational velocity $u^{*}$, then the visual servoing scheme controls the angular velocity $\omega$ so that the visual features $\mathbf{s}=\left(x_{f}, \theta_{m}\right)$ converge exponentially to $\mathbf{s}^{*}=(0,0)$. This ensures that the camera moves forward in the middle of the corridor. However it is important to note that since the camera is not mounted on the center of the wheelchair, we have to adjust the desired value of $\theta_{m}$ in order to ensure that the wheelchair (instead of the camera) remains in the middle of the corridor (see Section 6 for more details).

We refer the readers to [27] for further details on the control scheme design and the stability analysis. We consider the same visual features in order to initially design the wall avoidance task (detailed in the next Section) since they provide an efficient description of the orientation of the robot and its lateral position with respect to the corridor walls.

While the vanishing point $f_{t}$ can be estimated robustly from the scene 
by a method which is described in the following subsection, the median line is not visible in the image. This virtual line feature is then computed from the orientations $\theta_{l}$ and $\theta_{r}$ of the straight lines in the image related to the intersection of the walls and the floor of the corridor (see Figure 4). If the median of the corridor is projected onto the image as a straight line parametrized by $\left(\rho_{m}, \theta_{m}\right)$, we have

$$
\rho_{m}=x_{f} \cos \theta_{m}+y_{f} \sin \theta_{m} .
$$

Geometrically, in the 3D world, the lines related to the wall and the median of the corridor are parallel and coplanar. As a consequence, the corresponding lines in the image intersect at the vanishing point while respecting

$$
\theta_{m}=\arctan \left(\frac{\tan \theta_{l}+\tan \theta_{r}}{2}\right)
$$

Therefore, in order to estimate the median line feature parameters $\left(\rho_{m}, \theta_{m}\right)$, we have to extract the wall/floor boundary lines.
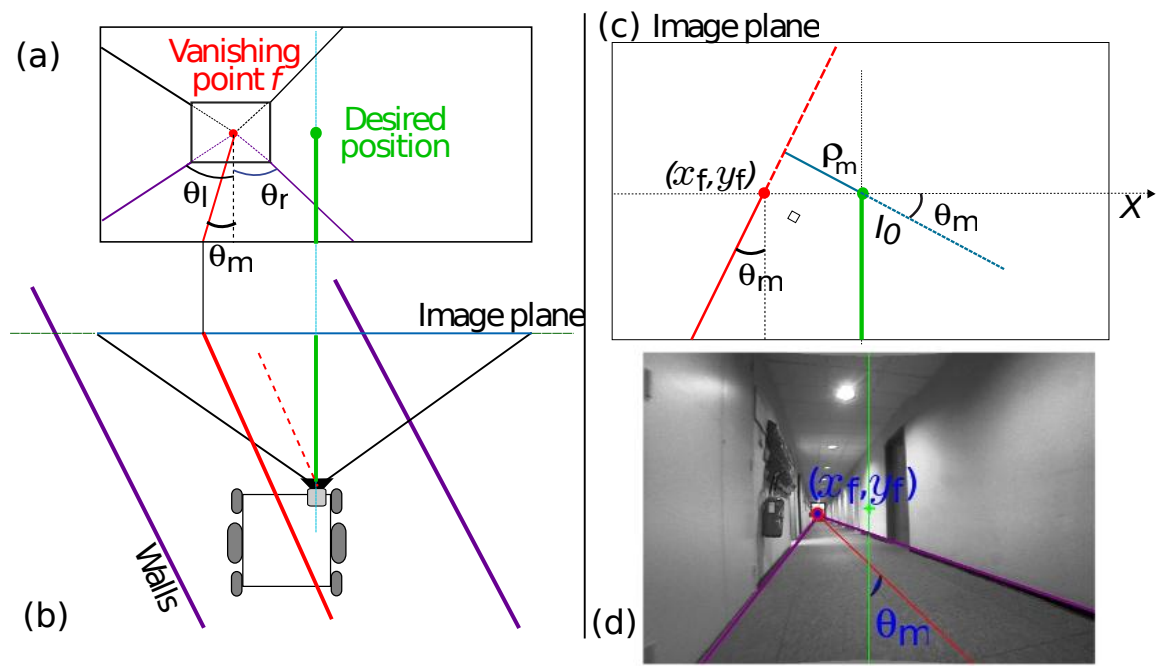

Figure 4: (a) Schematic representation of the visual features in the image along with its desired positions. The vanishing point is given in red. The angle $\theta_{m}$ is indicated with respect to the angles $\theta_{l}$ and $\theta_{r}$. (b) The median line of the corridor in red, (c) and its 2D projection in the image (parametrized by $\rho_{m}$ and $\theta_{m}$ ) (d) Feature extraction result on an generic corridor with the wall/floor boundaries detected along with the vanishing point. 


\subsection{Estimation of $x_{f}$}

For indoor scenes, estimating the vanishing point robustly and in real time is still an open area of research in computer vision. The vanishing point corresponds to the point where a significant number of straight lines may intersect. Therefore in the case of navigating through a corridor the problem is much easier to tackle.

We use the classical Gaussian sphere projection framework [29] [30], where the idea is to project onto this sphere, the set of detected non-vertical straight lines in the image. The point where a maximum number of these non-vertical lines intersect is chosen as the vanishing point. For extracting these nonvertical straight lines in the image, the Line Segment Detection (LSD) algorithm is used [31]. The algorithm is based on local gradient orientations in the image, from which major segments are detected. The detected segments are then classified into vertical lines and non-vertical lines and latter is used for estimating the vanishing point.

However, the main issue of the LSD algorithm is the removal of one line when the direction of the observed gradient changes. Thus, to increase the robustness, a dedicated merging process is applied to a group of segments that can be considered as a single straight line. For two segments, this process is done by taking into account the slope and extremities, and if they are close enough, they are merged to form a unique line. More details are given in [32].

Since the vanishing point $f_{t}$ is estimated at each frame during a sequence, a factor $\left.\alpha_{f} \in\right] 0,1[$ is introduced for temporal filtering as

$$
f_{t}=\alpha_{f} f_{t-1}+\left(1-\alpha_{f}\right) f_{t}
$$

in order to ensure a smooth variation of the resulting estimated vanishing point in the current frame. The value of $\alpha_{f}$ is empirically tuned and has been chosen for the experiments at $\alpha_{f}=0.1$. It can be postulated that since the temporal variation of the vanishing point is not very high as a wheelchair with a front facing camera moves forward in a corridor, the filtering process will be robust to variations in the factor $\alpha_{f}$.

\subsection{Estimation of $\theta_{m}$}

As we can see from Eqn. 5, $\theta_{m}$ is a function of $\theta_{l}$ and $\theta_{r}$ and therefore they have to be determined in order to estimate $\theta_{m}$. We can say that the angles $\theta_{l}$ and $\theta_{r}$ can be easily calculated if we have an accurate estimate 
of the wall/floor boundaries. Among the variety of techniques that have been proposed in the area of wall/floor boundary detection, in [33] wall/floor features are defined by the corners corresponding to the intersection of a vertical line and the floor plane. Whereas in [34], floor boundary is estimated by a dynamical Bayesian network model which is applied on each column of the image.

But we know that in an indoor corridor scene (see Figure 3) wall/floor boundary lines correspond to non-vertical lines that intersect the vanishing point. Therefore in our proposed scheme, a set of non-vertical lines are searched for along the image that correspond to the wall/floor boundary based on two criteria: the first one being that they contribute to the vanishing point and, the second one being that they cross the bottom extremities of most number of vertical lines. Again the non-vertical lines are detected and classified using the LSD algorithm. In order to minimize false positives and improve robustness, a maximal distance between the vertical line extremity and the vanishing line is defined. The result of the feature extraction process on a generic image can be seen in Figure $4 \mathrm{~d}$.

Then, from the angles $\theta_{l}$ and $\theta_{r}$, the value of $\theta_{m}$ is directly obtained using Eqn (5).

\subsection{Robustness of Feature Extraction}

Since the control scheme detailed in the following Section is driven by the visual features $x_{f}$ and $\theta_{m}$, the estimation of the features should be fairly robust with respect to different conditions occurring in a indoor corridor driving scene. We present a small driving sequence in Figure 5 with a variety of disturbances occurring in the scene. On each camera frame output the vanishing point is represented by the red cross (the intersection of all the lines in the image) and the median line of the corridor is represented by the

red line. The wall/floor boundaries are shown in purple. It can be observed in all the examples that the estimation of the features is quite accurate. Even when there is a direct occlusion with persons moving in front of the camera, the vanishing point position and the median line is correct. At the end of the corridor when there is very little composite wall/floor boundary the estimation is consistent, owing partly to the temporal filter used in the estimation of $x_{f}$. 


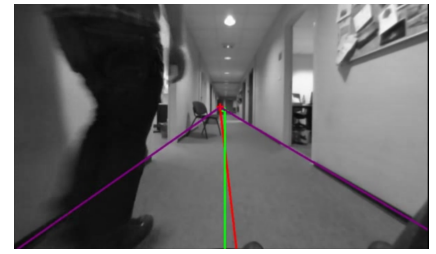

(a) Man walking on the side

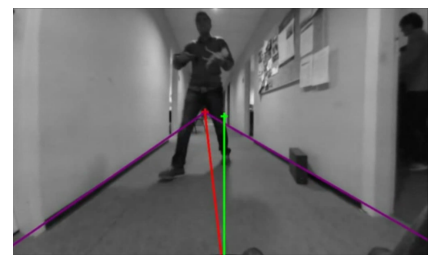

(d) Man walking in front

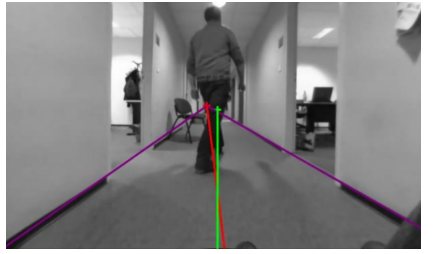

(b) Man walking in front

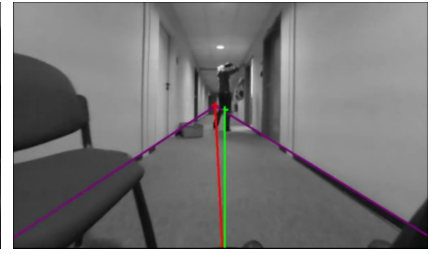

(e) Furniture in the scene

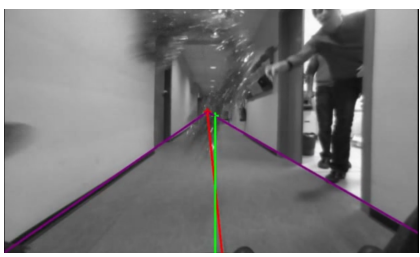

(c) Occlusion from random objects

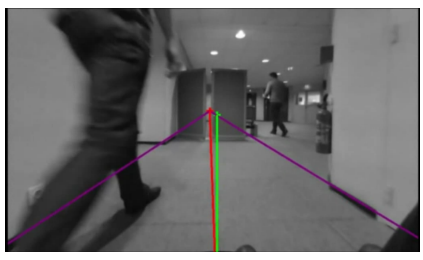

(f) End of corridor with man walking

Figure 5: The robustness of the feature extraction process with respect to different conditions and occlusions.

\section{Vision-based Assistance}

Let the user teleoperation output from the haptic joystick be denoted by $\mathbf{u}_{o p}=\left(u_{o p}, \omega_{o p}\right)$. The aim of the control scheme would be to then augment the user teleoperation in such a manner that it ensures that the user is the primary controller and the assistance is provided only when required. If the user is not in danger, then it is preferable to assign full control to the user. Therefore it is advantageous to design a wall collision avoidance task that progressively corrects the wheelchair trajectory as it comes closer to the walls. The following subsection summarizes a wall collision avoidance system, that is derived from the autonomous visual servoing task, and that can be blended with user output $\mathbf{u}_{o p}$ so that the trajectory can be corrected automatically when the user is in danger.

\subsection{Wall collision avoidance via Visual Servoing}

To ensure that the wheelchair does not hit the corridor walls, we propose to gradually activate the regulation of the visual features $\mathbf{s}=\left(x_{f}, \theta_{m}\right)$ to the desired values $\mathbf{s}^{*}=(0,0)$ when they leave a pre-fixed safe interval, namely $x_{f} \in\left[x_{f}^{s-}, x_{f}^{s+}\right]$ and $\theta_{m} \in\left[\theta_{m}^{s-}, \theta_{m}^{s+}\right]$, so that they go back inside these safe intervals. This concept of an interval that triggers the visual servoing was 
introduced in [35] and used in [36] to ensure the visibility of an organ section during remote ultrasound tele-echography.

Let $\mathbf{H}=\operatorname{Diag}\left(h_{x_{f}}, h_{\theta_{m}}\right)$ be a diagonal matrix that weights the visual error where $h_{x_{f}} \in[0 ; 1]$ and $h_{\theta_{m}} \in[0 ; 1]$ are varying weights respectively associated to the visual features $x_{f}$ and $\theta_{m}$. Owing to this definition we can propose the following control law that sets the system velocity $\mathbf{u}=(u, \omega)$ aimed at keeping the visual features inside their interval:

$$
\mathbf{u}=-\lambda\left(\mathbf{H J}_{s}\right)^{+} \mathbf{H e}
$$

where $\lambda>0$ is the control gain, $\mathbf{e}=\mathbf{s}-\mathbf{s}^{*}$ is the visual error and $\mathbf{J}_{s}$ is the image Jacobian that links the variation of the visual features to the robot control input such that $\dot{\mathbf{s}}=\mathbf{J}_{s} \mathbf{u}$. Whereas $\left(\mathbf{H J}_{s}\right)^{+}$is the Moore-Penrose pseudo-inverse of $\left(\mathbf{H} \mathbf{J}_{s}\right)$.

The image Jacobian $\mathbf{J}_{s}$ was determined in [26] and was formulated as follows:

$$
\mathbf{J}_{s}=\mathbf{L}_{s}{ }^{c} \mathbf{W}_{r}{ }^{r} \mathbf{J}_{r}
$$

with $\mathbf{L}_{s}$ being the interaction matrix that relates the variation of the visual features to the camera velocity screw. It is defined as

$$
\mathbf{L}_{s}=\left[\begin{array}{cccccc}
0 & 0 & 0 & x_{f} y_{f} & -1-x_{f}^{2} & y_{f} \\
\lambda_{\theta_{m}} \cos \left(\theta_{m}\right) & \lambda_{\theta_{m}} \sin \left(\theta_{m}\right) & -\lambda_{\theta_{m}} \rho_{m} & -\rho_{m} \cos \left(\theta_{m}\right) & -\rho_{m} \sin \left(\theta_{m}\right) & -1
\end{array}\right]
$$

with $\lambda_{\theta_{m}}=\cos \left(\theta_{m}\right) / h$ where $h$ is the distance of the camera from the floor (see the definition of ${ }^{c} \mathbf{t}_{r}$ in Section 3). By combining the expression for the robot Jacobian ${ }^{r} \mathbf{J}_{r}$ in Eqn. (3) and the expression for the velocity transformation matrix ${ }^{c} \mathbf{W}_{r}$ in Eqn. (2), we reformulate Eqn. (7) as

$$
\mathbf{J}_{s}=\left[\begin{array}{cc}
0 & 1+x_{f}^{2} \\
-\lambda_{\theta_{m}} \rho_{m} & -\lambda_{\theta_{m}} l \cos \left(\theta_{m}\right)+\lambda_{\theta_{m}} w \rho_{m}+\rho_{m} \sin \left(\theta_{m}\right)
\end{array}\right]=\left[\begin{array}{c}
\mathbf{J}_{x_{f}} \\
\mathbf{J}_{\theta_{m}}
\end{array}\right]
$$

In the definition of the weight matrix $\mathbf{H}=\operatorname{Diag}\left(h_{x_{f}}, h_{\theta_{m}}\right)$, a zero weight means that the related visual feature is not regulated by the visual servoing. The matrix $\mathbf{H}$ allows then to add or remove any visual feature in the control law when desired and can totally deactivate the visual servoing when $\mathbf{H}$ is zero. In order to gradually activate the wall avoidance task when a visual feature leaves its safe interval, we propose to define the weight related to $x_{f}$ 
by the following smooth function:

$$
h_{x_{f}}\left(x_{f}\right)=\left\{\begin{array}{cl}
\left(1-\cos \left(\pi \frac{x_{f}-x_{f}^{s-}}{x_{f}^{-}-x_{f}^{s-}}\right)\right) / 2 & \text { if } x_{f}^{-} \leq x_{f} \leq x_{f}^{s-} \\
0 & \text { if } x_{f}^{s-}<x_{f}<x_{f}^{s+} \\
\left(1-\cos \left(\pi \frac{x_{f}-x_{f}^{s+}}{x_{f}^{+}-x_{f}^{s+}}\right)\right) / 2 & \text { if } x_{f}^{s+} \leq x_{f} \leq x_{f}^{+} \\
1 & \text { otherwise }
\end{array}\right.
$$

where $\left[x_{f}^{s-}, x_{f}^{s+}\right]$ is a safe interval within which the visual servoing task is fully deactivated (for the feature $x_{f}$ ). Whereas $\left[x_{f}^{-}, x_{f}^{+}\right]$is the unsafe interval whose fixed limits should never be overcome owing to the visual servoing scheme. A similar expression for $h_{\theta_{m}}$ can also be proposed. The corresponding function evolution is shown in Figure 6. We can note that each weight is zero in the safe interval and increases up to 1 if the related feature comes close to the tolerated interval limits. In this way, the wall avoidance task is gradually activated when the weight related to the visual feature is increasing.

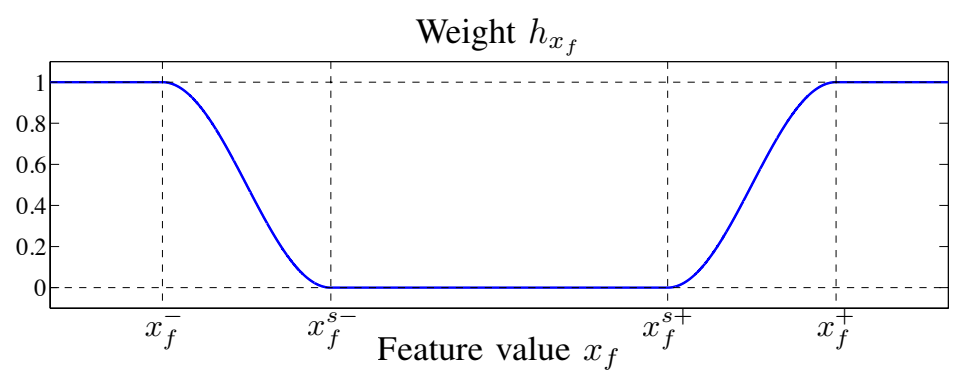

Figure 6: Weighting function $h_{x_{f}}$ defined for feature $x_{f}$. The weight is zero in the safe interval and increases smoothly up to 1 at the borders of the tolerated interval $\left[x_{f}^{-}, x_{f}^{+}\right]$(a similar function is used to define the weight $h_{\theta_{m}}$ with intervals $\left[\theta_{m}^{-}, \theta_{m}^{+}\right]$and $\left.\left[\theta_{m}^{s-}, \theta_{m}^{s+}\right]\right)$.

The three conditions to obtain a continuous behaviour of the control law (6) are that $\mathbf{J}_{s}, \mathbf{H}$ and the pseudo-inverse of $\mathbf{H J}_{s}$ remain continuous. The two first conditions are valid according to the form of (8) and the weight definition (9). However, the pseudo-inverse is not continuous since the rank of $\mathbf{H J}_{s}$ could switch from zero, if all features are in their safe intervals, to 1 when only one feature leaves its interval or even 2 (full rank) when the two features are outside their safe intervals. To avoid discontinuities in the control we propose therefore to replace like in [37] the Moore-Penrose pseudo-inverse operator ${ }^{+}$by the continuous pseudo-inverse operator $\oplus \mathbf{H}$ introduced in the 
framework of varying-feature-set [38]. This operator allows the inversion of a matrix say $\mathbf{J}$ weighted by a diagonal matrix say $\mathbf{H}$ by applying the following definition:

$$
\mathbf{J}^{\oplus \mathbf{H}}=\sum_{\mathcal{P} \in \mathfrak{B}(k)}\left(\prod_{i \in \mathcal{P}} h_{i}\right)\left(\prod_{i \notin \mathcal{P}}\left(1-h_{i}\right)\right) \mathbf{J}_{\mathcal{P}}^{+}
$$

where $\mathbf{J}$ is a matrix of size $(k \times n), \mathbf{H}$ is a diagonal activation matrix of size $(k \times k)$ whose components $\left(h_{i}\right)_{i \in[1 \ldots k]}$ are included in the interval $[0,1] \cdot \mathfrak{B}(k)$ is the set of all the combinations formed by the integers belonging between 1 and $k$ (for example $\mathfrak{B}(2)=\{\emptyset,\{1\},\{2\},\{1,2\}\}$ ). $\mathcal{P}$ is any element of this set and $\mathbf{J}_{\mathcal{P}}=\mathbf{H}_{0} \mathbf{J}$ with $\mathbf{H}_{0}$ being a $(k \times k)$ diagonal matrix whose diagonal component $(i, i)$ is equal to 1 if $i \in \mathcal{P}$ and to 0 otherwise. All the theoretical bases including the proof of continuity of this inversion operator are presented in [38]. By applying this operator (with $k=2$ ), the continuous inversion of the image Jacobian $\mathbf{J}_{s}$ activated by the weight matrix $\mathbf{H}$ can be obtained as

$$
\mathbf{J}_{s}^{\oplus \mathbf{H}}=h_{x_{f}}\left(1-h_{\theta_{m}}\right)\left[\begin{array}{c}
\mathbf{J}_{x_{f}} \\
\mathbf{0}_{1 \times 2}
\end{array}\right]^{+}+\left(1-h_{x_{f}}\right) h_{\theta_{m}}\left[\begin{array}{c}
\mathbf{0}_{1 \times 2} \\
\mathbf{J}_{\theta_{m}}
\end{array}\right]^{+}+h_{x_{f}} h_{\theta_{m}} \mathbf{J}_{s}^{+}
$$

We can note that if both the weights of $\mathbf{H}$ are equal to 1 (full activation of the wall avoidance task) then the matrix $\mathbf{J}_{s}^{\oplus \mathbf{H}}$ is exactly equal to $\left(\mathbf{H J}_{s}\right)^{+} \mathbf{H}$ and we have the same equality if all the weights are zero (deactivation of the wall avoidance task).

Hence the control law (6) can be replaced by the following control law ensuring the wall avoidance task with a continuous behaviour:

$$
\mathbf{u}=-\lambda \mathbf{J}_{s}^{\oplus} \mathbf{H} \mathbf{e} .
$$

\subsection{Fusion of User and Robot Control}

In order to create a semi-autonomous system that prevents the user from hitting the lateral walls while he is manually driving the wheelchair, manual control has to be fused with the wall collision avoidance task. The above visual task constraints only the DOFs that regulates the activated features into their safe interval. It is then possible to control the remaining DOFs using the well established redundancy formalism [39]. Therefore when both the features have returned to their safe intervals, all the DOFs are fully available for the manual control since the visual task is fully deactivated.

This also means that the desired features $\mathbf{s}^{*}$ will never be reached (which is not a problem since the objective of the visual servoing is only to bring 
them back in their safe intervals). The wall avoidance task can therefore be blended with user teleoperation output $\mathbf{u}_{o p}$ from the haptic joystick so that

$$
\mathbf{u}=-\lambda \mathbf{J}_{s}^{\oplus} \mathbf{H} \mathbf{e}+\mathbf{P}_{\oplus} \mathbf{u}_{o p}
$$

where $\mathbf{P}_{\oplus}=\mathbb{I}_{2}-\mathbf{J}_{s}^{\oplus \mathbf{H}} \mathbf{J}_{s}$ is the projection operator presented in [37]. The projection operator $\mathbf{P}_{\oplus}$ projects the components of $\mathbf{u}_{o p}$ (which is the desired user teleoperation velocity output) onto the null space of the wall collision avoidance task so that manual control does not disturb the visual task which has higher priority when in danger. But when both features are in their safe intervals, the visual task is fully deactivated and the user has full control as $\mathbf{P}_{\oplus}=\mathbb{I}_{2}$. We then obtain a smooth and progressive trajectory correction framework that is only activated in case of danger.

\subsection{Design of Haptic Guidance}

Such a semi-autonomous assistive system which provides adaptive assistance in the form of wall collision avoidance is an extremely helpful tool for wheelchair users. But in the case of users suffering from severe motor disabilities and visual/cognitive impairments, automatic trajectory correction may reduce the quality of experience, mainly if the user is not able to immediately perceive the danger. In order to communicate the objective of the system to the user and to notify the user of his unsafe trajectory, we have to design a joystick force feedback mechanism that intuitively guides the user out of this unsafe trajectory. Thus we have to set an optimal mapping function that maps the assistance provided to the force fed back for guidance.

As mentioned in Sec. 3, we assume a 2-DOF haptic joystick having the capability of assigning forces in the $x_{F}$ and $y_{F}$ directions with $\mathbf{F}=\left(f_{x}, f_{y}\right)^{T}$ as illustrated in Figure 2d. If we observe Equation (13), it is possible to identify that the projection operator $\mathbf{P}_{\oplus}=\mathbb{I}_{2}-\mathbf{J}_{s}^{\oplus \mathbf{H}} \mathbf{J}_{s}$ represents the amount of control that the user has at a particular configuration.

Naturally, if all the visual features are in their safe intervals, $\mathbf{J}_{s}^{\oplus \mathbf{H}} \mathbf{e}$ is null which makes $\mathbf{P}_{\oplus}=\mathbb{I}_{2}$, and the system does not realize any control. At this point the user has full control over the motion (as there is no risk of collision). As soon as the features leave their safe intervals, $\mathbf{J}_{s}^{\oplus \mathbf{H}}$ becomes non-zero and the system progressively takes up some control over the motion. When both the features are constrained by the visual task we have $\mathbf{J}_{s}^{\oplus \mathbf{H}} \mathbf{J}_{s}=\mathbb{I}_{2}$ and at this point the system has full control. 


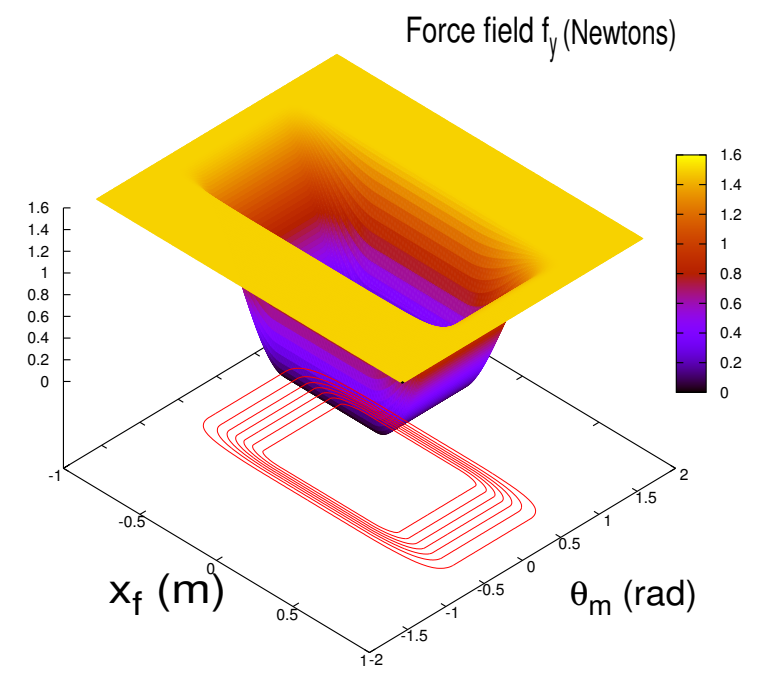

Figure 7: Model variation in the absolute value of $f_{y}$ with respect to the variation in visual features $x_{f}$ and $\theta_{m}$. Generated using $x_{f}^{s-}=-0.3 \mathrm{~m}, x_{f}^{s+}=0.3 \mathrm{~m}, x_{f}^{-}=-0.9 \mathrm{~m}, x_{f}^{+}=$ $0.9 \mathrm{~m}, \theta_{m}^{s-}=-0.5 \mathrm{rad}, \theta_{m}^{s+}=0.5 \mathrm{rad}, \theta_{m}^{-}=-0.8 \mathrm{rad}, \theta_{m}^{+}=0.8 \mathrm{rad}$. The upper bound $f_{\max }$ was set at $1.5 \mathrm{~N}$ and $\alpha$ at 1 . We can observe the progressive increase in force from 0 to 1.5 as the visual features leave their safe interval.

If the maximum exertable force at nominal position by the haptic joystick is denoted by $f_{\max }$ (expressed in Newtons), we can determine the force feedback $\mathbf{F}$ as

$$
\mathbf{F}=\alpha \mathbf{J}_{s}^{\oplus \mathbf{H}} \mathbf{J}_{s} \mathbf{F}_{\text {max }}
$$

where $\mathbf{F}_{\max }=\left( \pm f_{\max }, \pm f_{\max }\right)^{T}$ and $\alpha$ is a factor required to normalize the force so that it can be handled by the user. The sign of the $x_{F}$ and $y_{F}$ components in $\mathbf{F}_{\text {max }}$ depends on the visual features $x_{f}$ and $\theta_{m}$ at a particular instant. We have

$$
\mathbf{F}_{\text {max }}=\left\{\begin{array}{cl}
\left(-f_{\text {max }},-f_{\text {max }}\right)^{T} & \text { if } x_{f} \leq x_{f}^{*} \text { and } \theta_{m}>\theta_{m}^{*} \\
\left(-f_{\text {max }}, f_{\text {max }}\right)^{T} & \text { if } x_{f} \leq x_{f}^{*} \text { and } \theta_{m} \leq \theta_{m}^{*} \\
\left(f_{\max },-f_{\text {max }}\right)^{T} & \text { if } x_{f}>x_{f}^{*} \text { and } \theta_{m}>\theta_{m}^{*} \\
\left(f_{\max }, f_{\text {max }}\right)^{T} & \text { if } x_{f}>x_{f}^{*} \text { and } \theta_{m} \leq \theta_{m}^{*}
\end{array}\right.
$$

Therefore, when $\mathbf{J}_{s}^{\oplus \mathbf{H}}$ is null, no force is applied on the joystick. As the matrix becomes non-zero and eventually reaches rank 2 , the force $\mathbf{F}$ increases adaptively and reaches $\alpha \mathbf{F}_{\text {max }}$. At this point the control system has full 
control and a maximum force is applied on the joystick to notify the user of his dangerous trajectory. A model force variation in the absolute value of $f_{y}$ is shown in Figure 7 with respect to variations in the visual features.

It has to be noted that the force applied modifies the position of the joystick at a particular configuration (in a direction which corrects the motion of the wheelchair) thereby affecting $\mathbf{u}_{o p}$. This does not affect the system stability since the user output is explicitly considered in the control design. This also ensures that a minimal automatic correction will be required from the system for collision avoidance thus leading to a higher quality of experience.

But it is also possible to design a system that passively guides the user by taking into account the force fed back $\mathbf{F}$ as well as the force applied by the user $\mathbf{F}^{*}$ against the feedback force. In that case, we have

$$
\mathbf{u}=-\lambda \mathbf{J}_{s}^{\oplus} \mathbf{H} \mathbf{e}+\mathbf{P}_{\oplus}\left(\mathbf{u}_{o p}-\mathbf{u}_{f}\right)
$$

where $\mathbf{u}_{f}$ represents the velocity that would be transmitted to the wheelchair motion control system as a result of the feedback force $\mathbf{F}$ and the user force $\mathbf{F}^{*}$. In this study we refrain to active feedback as the preliminary tests have concluded that it is more intuitive and helpful in guiding a user than a passive feedback scheme.

\section{Experimental Analysis}

\subsection{Test Setup}

An extensive analysis was carried out in order to assess the effectiveness of the assistive mechanism and the feedback guidance scheme as a modular and efficient tool for safe corridor navigation. An off-the-shelf wheelchair manufactured by You-Q which was robotised using the ROS middleware [40] was used for testing. A front facing Raspberry Pi camera module with an $85^{\circ}$ field of view ( $f o v)$ was rigidly fixed on the left handle as shown in Figure 8. It was also coarsely calibrated with $h=0.8 \mathrm{~m}, w=0.32 \mathrm{~m}$ and $l=0.4 \mathrm{~m}$. A traditional joystick as well as a Phantom Omni haptic device were connected to the wheelchair control system in order to compare the proposed control scheme with and without force feedback. The haptic joystick had a maximum exertable force $f_{\max }=3.3 \mathrm{~N}$ at nominal position.

Prior to the feature extraction process the images were rectified against distortions from the camera lens. The feature extraction and the control scheme computation were performed using the ViSP [41] software. The visual servoing algorithm was performed on a Core i7 Laptop connected to 

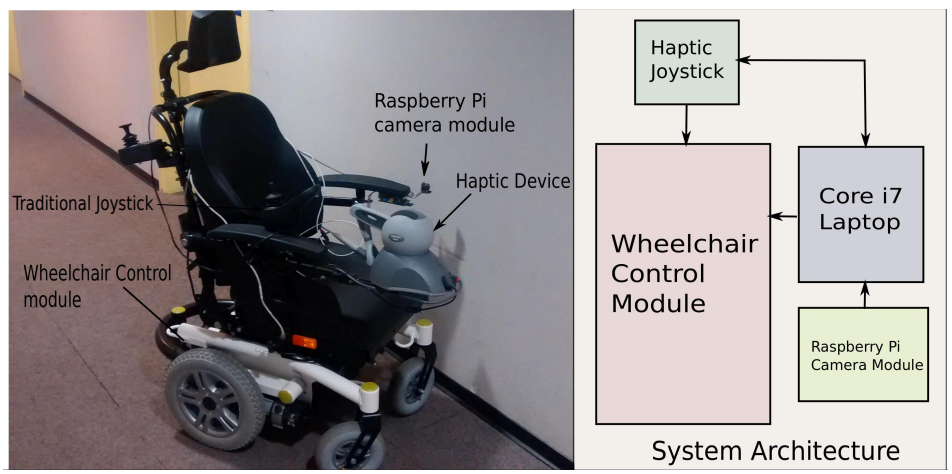

Figure 8: Wheelchair test platform

the wheelchair control system using Ethernet. For ground truth estimation and visualisation purposes, the wheelchair had been equipped with a laser range finder. This laser range finder was used neither in the visual feature extraction process nor in the control law. It only acted as a validation tool.

An analysis of the convergence and stability of the visual servoing task for corridor following is presented in [27]. Experimental validation is also presented where a wheelchair equipped with a single monocular camera is used to autonomously follow a corridor using the features detailed in Section 4.

Now to be able to perform experiments using the control law proposed in the present work, the parameters to be determined include the boundaries of the activation intervals (i.e $x_{f}^{+}, x_{f}^{s+}, x_{f}^{-}, x_{f}^{s-}, \theta_{m}^{+}, \theta_{m}^{s+}, \theta_{m}^{-}$and $\theta_{m}^{s-}$ ), the control gain $\lambda$ and the factor $\alpha$ (see Eqn. (14)).

$x_{f}^{+}, x_{f}^{s+}, x_{f}^{-}, x_{f}^{s-}$ are directly dependent to the field of view (fov) of the camera. In our setup, the maximum and minimun possible values of $x_{f}$ are respectively equal to $\tan (f \circ v / 2) \approx 0.916$ and $-\tan ($ fov $/ 2) \approx-0.916$. Under these constraints, we chose $x_{f}^{+}=0.9$ and $x_{f}^{-}=-0.9$ to ensure the visibility of the vanishing point. As the visual feature extraction algorithm needs to detect both floor/wall boundary lines, we chose $x_{f}^{s+}=0.3$ and $x_{f}^{s-}=-0.3$, to ensure the visibility of these features.

$\theta_{m}^{+}, \theta_{m}^{s+}, \theta_{m}^{-}$and $\theta_{m}^{s-}$ are directly dependent to the width of the corridor used during the experimentation. The width of the corridor $W_{\text {cor }}$ can be estimated online during the feature extraction process using $W_{\text {cor }} \approx$ $h\left(\tan \left(\theta_{l}\right)+\tan \left(\theta_{r}\right)\right)$. Then we define $\operatorname{margin}_{l}$ and $\operatorname{margin}_{r}$ as the approximate margin between the walls and the camera. margin $_{a c t}$ is defined as 


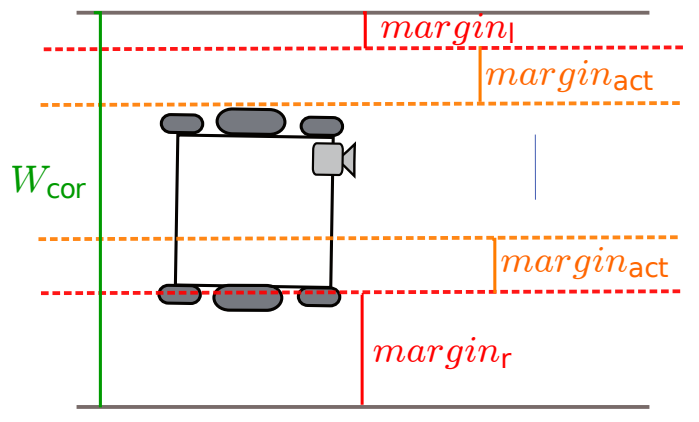

Figure 9: Margin definition

depicted in Figure 9. We can then determine

$$
\begin{aligned}
\theta_{m}^{+} & =\arctan \left(\frac{W_{\text {cor }}-2 \text { margin }_{r}}{h}\right) \\
\theta_{m}^{s+} & =\arctan \left(\frac{W_{\text {cor }}-2 \text { margin }_{r}-2 \text { margin }_{\text {act }}}{h}\right) \\
\theta_{m}^{-} & =\arctan \left(\frac{2 \text { margin }_{l}-W_{\text {cor }}}{h}\right) \\
\theta_{m}^{s-} & =\arctan \left(\frac{2 \text { margin }_{l}+2 \text { margin }_{\text {act }}-W_{\text {cor }}}{h}\right)
\end{aligned}
$$

In our experiments, we choose $\operatorname{margin}_{l}=0.15 \mathrm{~m}, \operatorname{margin}_{r}=0.60 \mathrm{~m}$ and margin $_{\text {act }}=0.30 \mathrm{~m}$ and $W_{\text {cor }}$ is estimated using the camera at initialisation and is kept constant for the rest of the experiments. This is done in order to negate the effects of a varying $\theta_{m}$ interval. Finally the gain $\lambda$ was empirically determined and set at 0.3 and the normalizing factor $\alpha$ at 0.7 so that the maximum force experienced by the user is capped at $2.2 \mathrm{~N}$.

For analysing the full potential of the control system, a non-disabled user was asked to steer the wheelchair along corridors. Initial tests were carried out using a traditional joystick with no feedback in order to assess the efficiency of the automatic trajectory correction mechanism. Later tests were carried out using the haptic joystick in order to gauge the effects of force feedback on trajectory correction and user experience. The results presented here pertains to experiments carried out inside the Inria building in Rennes, France. The visual task is activated as soon as the wheelchair starts the motion and it is switched off as the user reaches the end of the corridor. 


\subsection{Semi-autonomous navigation without force feedback}

As the user drives the wheelchair manually in the corridor, Figures 12 and 13 respectively show the variation of the visual feature $x_{f}$ and $\theta_{m}$ plotted along with their activation factors $h_{x_{f}}$ and $h_{\theta_{m}}$. Figure 14 presents the evolution of the user teleoperation $\left(\mathbf{u}_{\mathbf{o p}}\right)$ and final system $(\mathbf{u})$ velocities during the experimentation with the translational components $u$ and $u_{o p}$ (top) and the angular components $\omega$ and $\omega_{o p}$ (bottom). Each plot is divided into sections for easier analysis. Figure 10 presents outputs of the wheelchair camera together with visualization of the position of the wheelchair in the corridor from the laser range finder at different key points during the experimentation. On each camera output, the blue/green line represents the estimated middle line of the corridor and the cross the estimated position of the vanishing point (which is also the intersection of the lines plotted in the images). The color of the line (cross) refers to the value of $h_{\theta_{m}}\left(h_{x_{f}}\right)$, and it is green when $h_{\theta_{m}}=0\left(h_{x_{f}}=0\right)$ and blue when $h_{\theta_{m}}>0\left(h_{x_{f}}>0\right)$. The red and orange lines correspond to the boundary of $\theta_{m}$ according to (9). On each output of the laser range finder, the position of the wheelchair has been depicted in red with an arrow depicting the orientation. Finally for a better comprehension of the configuration of the wheelchair in the corridor, the relative position and heading of the wheelchair with respect to the median line of the corridor are shown in Figures 11a and 11b.

Note that for this experiment we obtain, from Eqns. (17) and (18), $\theta_{m}^{+}=$ $0.3 \mathrm{rad}, \theta_{m}^{s+}=-0.2 \mathrm{rad}, \theta_{m}^{-}=-0.55 \mathrm{rad}$ and $\theta_{m}^{s-}=-0.8 \mathrm{rad}$. Also the video stream from the camera corresponded to a resolution of $808 \times 480$ pixels with a frame rate of 15 fps.

In Figure 14, in parts A, C, E and G, we can then observe that $u=u_{o p}$ and $\omega=\omega_{o p}$ since $h_{x_{f}}=0$ and $h_{\theta_{m}}=0$. This is owing to the fact that $x_{f}$ remains in the $\left[x_{f}^{s-}, x_{f}^{s+}\right]$ interval and $\theta_{m}$ remains in the $\left[\theta_{m}^{s-}, \theta_{m}^{s+}\right]$ interval (see Figures 12 and 13). The user has then full control of the wheelchair in both translation and rotation. In Figure 10, frame 15 corresponds to part A and frame 160 corresponds to part E. On the laser range finder, we can observe that the wheelchair is in the middle of the corridor with a low orientation angle. In such a case, there is no risk of collision with the wall and the user has full control over the wheelchair motion.

Whereas in parts B,D and F, $h_{x_{f}}>0$ and $h_{\theta_{m}}>0$. We can then observe that $u \neq u_{o p}$ and $\omega \neq \omega_{o p}$. If we observe Figure 10c (Section B, frame 69), the wheelchair is close to the right wall with a low orientation angle. There is then a risk of collision if the user tries to turn right. Therefore, the control 
Section A : Frame 15

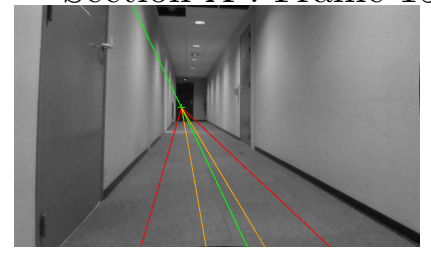

a) Wheelchair camera

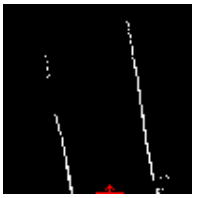

b) Ground truth

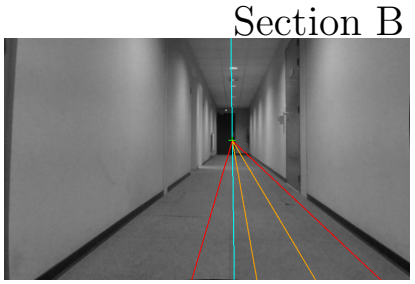

c) Wheelchair camera

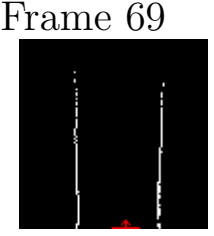

d) Ground truth

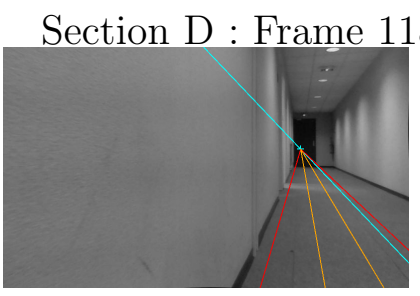

e) Wheelchair camera

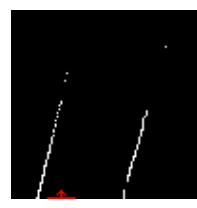

f) Ground truth

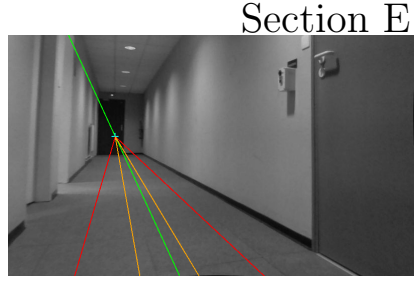

g) Wheelchair camera

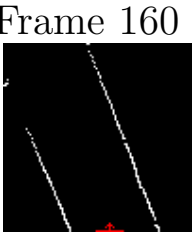

h) Ground truth

Figure 10: Wheelchair camera frames and ground truth during the experimentation

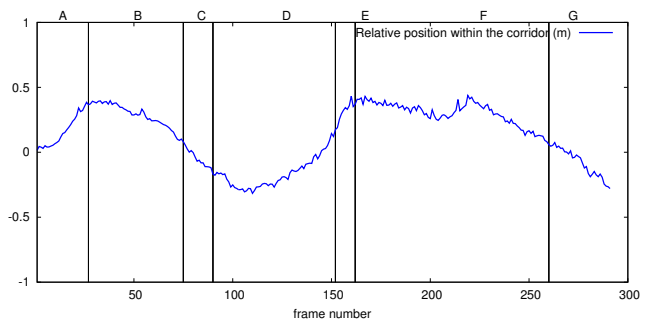

(a)

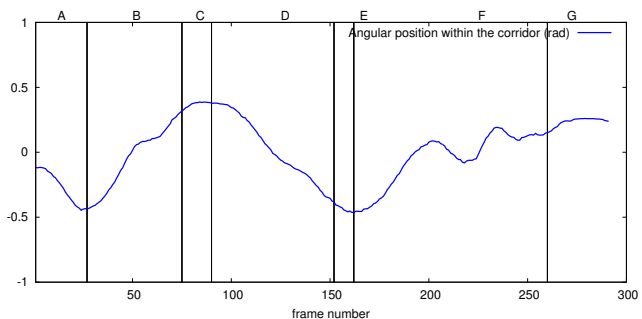

(b)

Figure 11: (a). The relative position of the wheelchair with respect to the median of the corridor. (b). The heading of the wheelchair with respect to the median of the corridor. Plots obtained by initially reconstructing the laser scan output using Hough transform in order to discriminate the longest lines in the image. The relative position and orientation can be then extracted be from the position and orientation of the two detected lines.

law is activated and augments $u_{o p}$ and $\omega_{o p}$ to avoid wall collision. Moreover in part D (see Figure 10e, related to the frame 118), the wheelchair is close to the left wall and oriented toward the wall. There is an imminent risk of collision. The translation velocity $u$ is reduced to avoid collision and the rotation velocity $\omega$ is forced to a negative value to get further from the wall.

We can observe that during the experimentation, $x_{f}$ and $\theta_{m}$ were respec- 


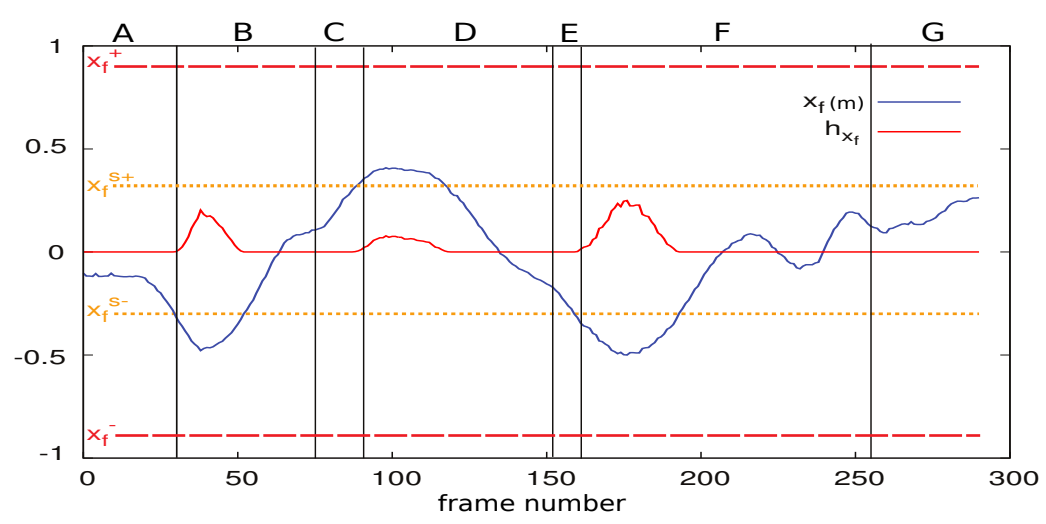

Figure 12: Evolution of $x_{f}$ along with its activation factor $h_{x_{f}}$.

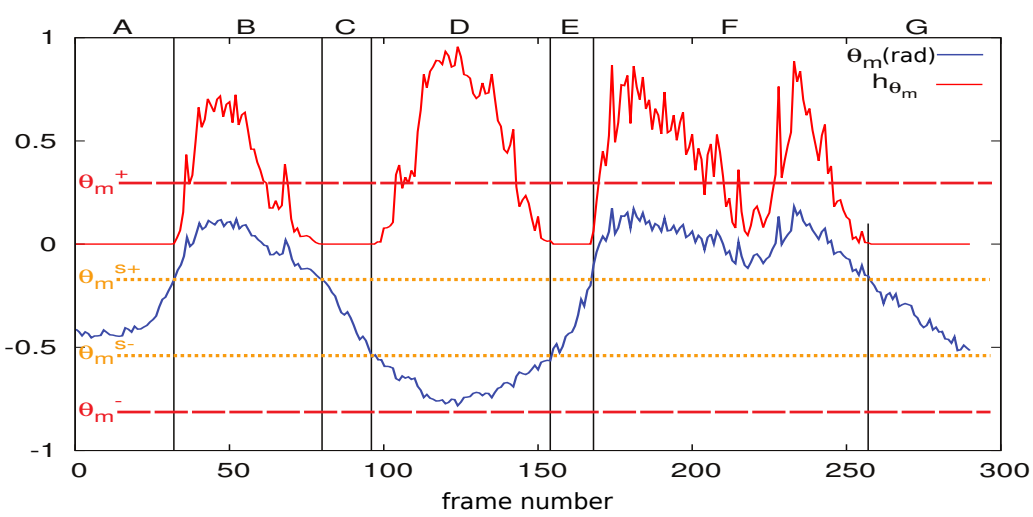

Figure 13: Evolution of $\theta_{m}$ along with its activation factor $h_{\theta_{m}}$.

tively forced by the visual servoing to remain in the interval $\left[x_{f}^{s-}, x_{f}^{s+}\right]$ and $\left[\theta_{m}^{s-}, \theta_{m}^{s+}\right]$ as expected. The behaviour of the system demonstrates that as the wheelchair gets closer to the corridor walls, the wall avoidance visual task is progressively activated thereby forcing the visual features into their safe intervals. This effectively steers the wheelchair away from the walls and into safety.

\subsection{Semi-autonomous navigation with force feedback}

A similar trial was carried out with force feedback added into the loop as explained in Section 5.3. In this case, we obtain $\theta_{m}^{+}=0.5 \mathrm{rad}, \theta_{m}^{s+}=-0.1$ 

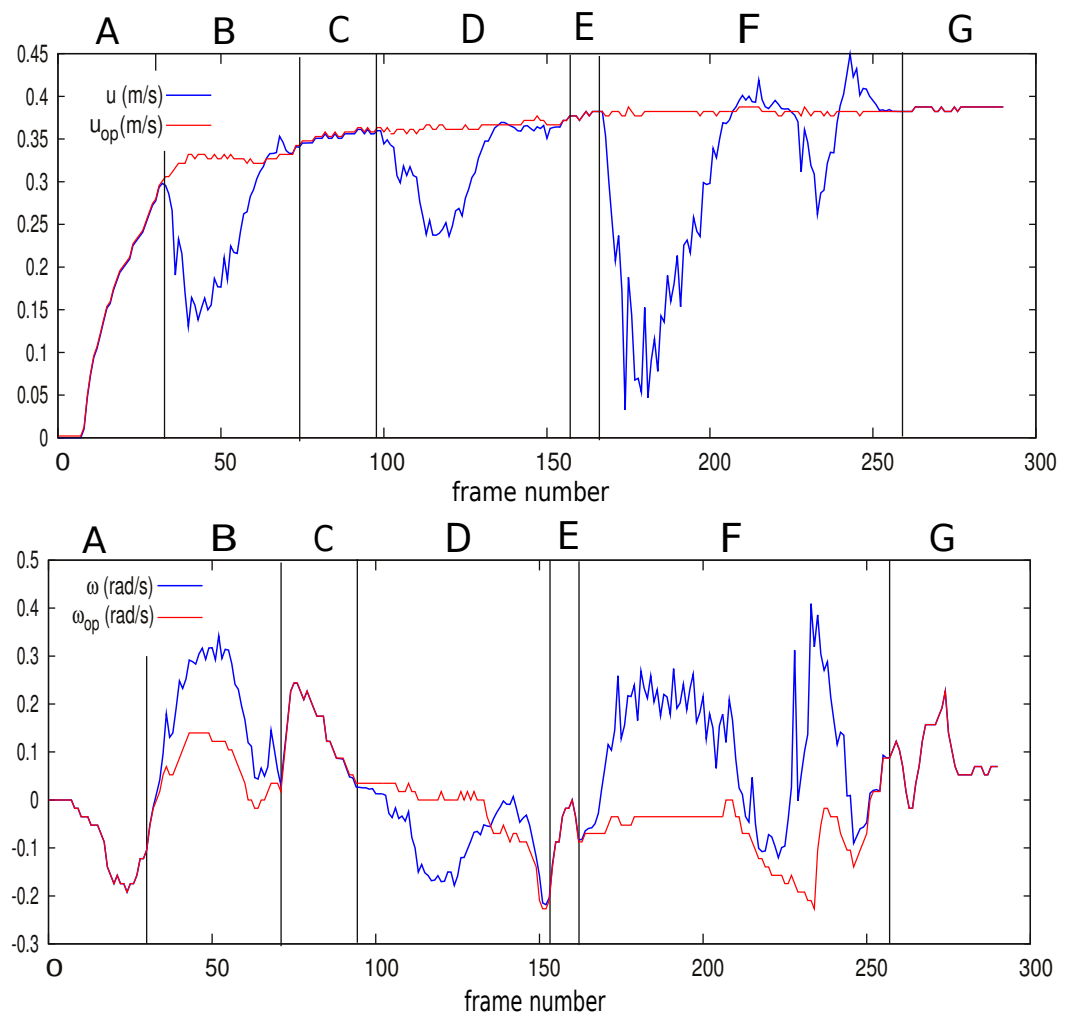

Figure 14: Evolution of $u$ along with $u_{o p}$ and $\omega$ along with $\omega_{o p}$.

$\mathrm{rad}, \theta_{m}^{-}=-0.6 \mathrm{rad}$ and $\theta_{m}^{s-}=-1 \mathrm{rad}$ and the video stream from the camera corresponded to a resolution of 640x480 pixels with a frame rate of $90 \mathrm{fps}$.

The evolution in the visual features $x_{f}$ and $\theta_{m}$ along with their respective activation factors $h_{x_{f}}$ and $h_{\theta_{m}}$ are plotted similarly in Figures 17 and 18. The variations in the forces $f_{x}$ and $f_{y}$ transmitted to the haptic joystick are given in Figure 19. Figure 20 represents the user teleoperation and final system velocity components namely $u_{o p}$ with $u$ and $\omega_{o p}$ with $\omega$. Figure 21 shows the variation in the automatic correction applied by the system in order to avoid wall collisions denoted by say $\mathbf{u}_{\mathbf{c}}=\left[u_{c}, \omega_{c}\right]^{T}$ where $\mathbf{u}_{\mathbf{c}}=-\lambda \mathbf{J}_{s}^{\oplus \mathbf{H}} \mathbf{e}$. Each plot is discretized into nine parts (A-I) for analysis. Finally, camera frames at specific points during the experiment are shown in Figures 15 and 16. The extracted features are overlayed onto each image as explained in the previous Section. 


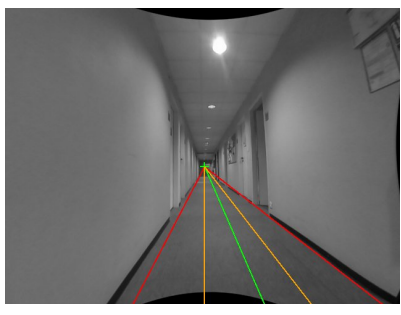

(a) Sec. A, Frame 213

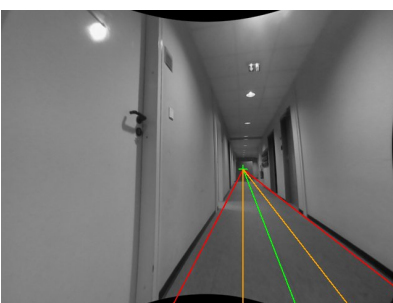

(b) Sec. E, Frame 445

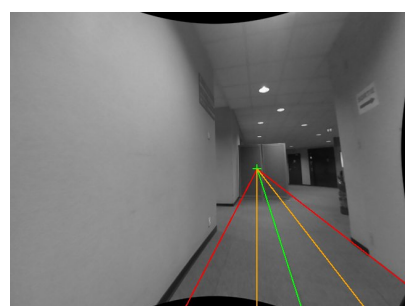

(c) Sec. I, Frame 1090

Figure 15: Camera frames at parts A, E and I where assistance and force feedback are not provided by the system.

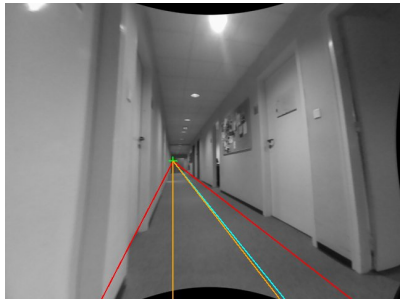

(a) Sec. B, Frame 256

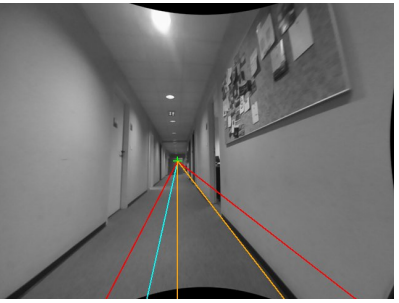

(b) Sec. D, Frame 382

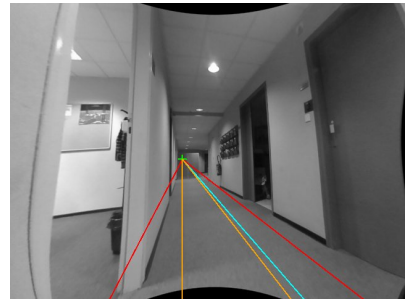

(c) Sec. F, Frame 643

Figure 16: Camera frames at parts B, D and $\mathrm{F}$ where assistance and force feedback are provided by the system. 


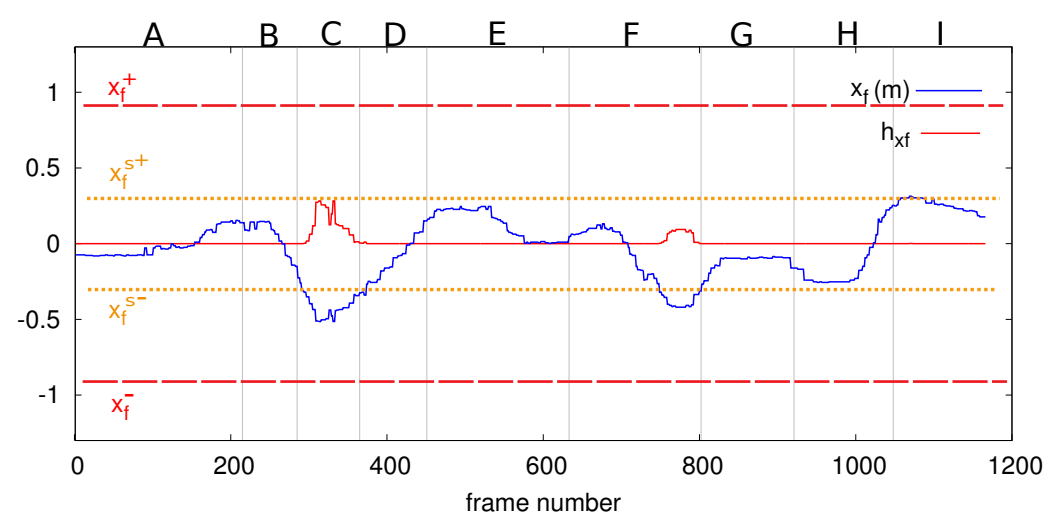

Figure 17: Visual feature $x_{f}$ along with activation factor $h_{x_{f}}$.

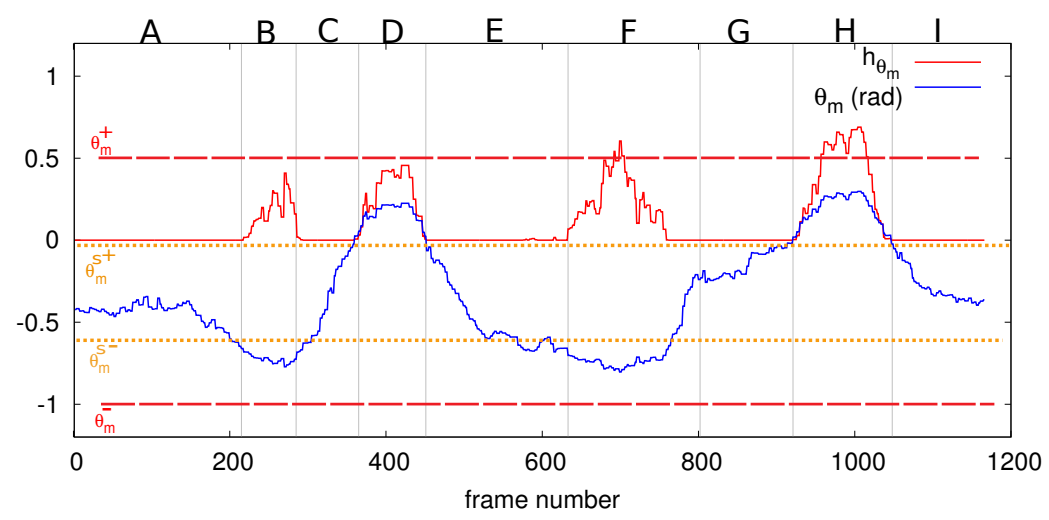

Figure 18: Visual feature $\theta_{m}$ along with activation factor $h_{\theta_{m}}$.

It can be seen that in parts A, E, G and I the visual features are within their safe intervals and no force is applied on the joystick. Also, the translational and rotational velocities $u_{o p}$ and $\omega_{o p}$ transmitted by the user completely match the final system velocities $u$ and $\omega$. This means that the user has full control over the motion since there is no risk of collision with the walls. Figure 15 shows the camera frames at parts A, E and I. Again at this point it can be said that the wheelchair is nearly in the middle of the corridor and is executing a safe motion.

Whereas in parts B, C, D, F and H, either one or both visual features are outside their safe intervals that leads to the activation of trajectory correction with force feedback. The camera frames at parts B, D and F are illustrated in Figure 16. Clearly the wheelchair is close to the walls: this can be observed 


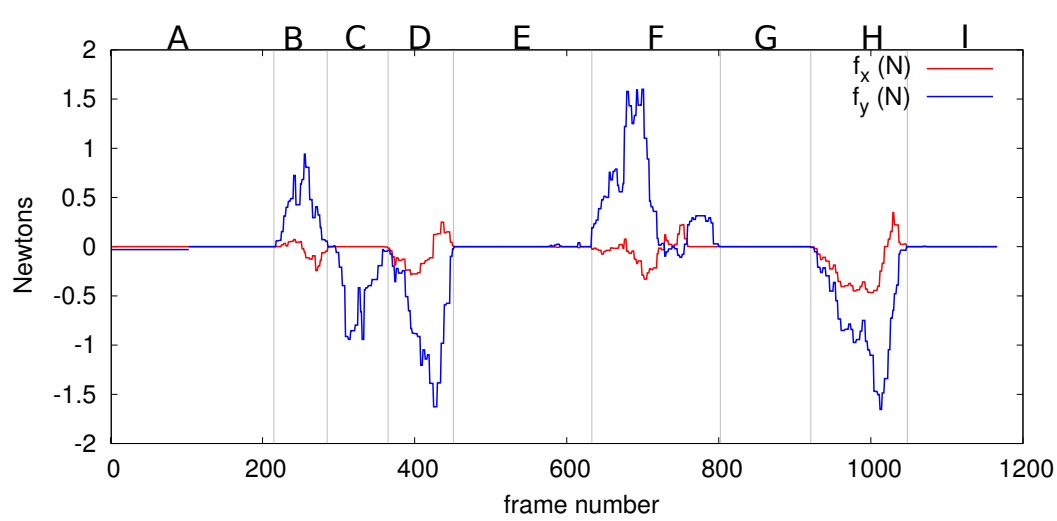

Figure 19: Evolution of the force applied on the haptic device in $x$ and $y$ directions.
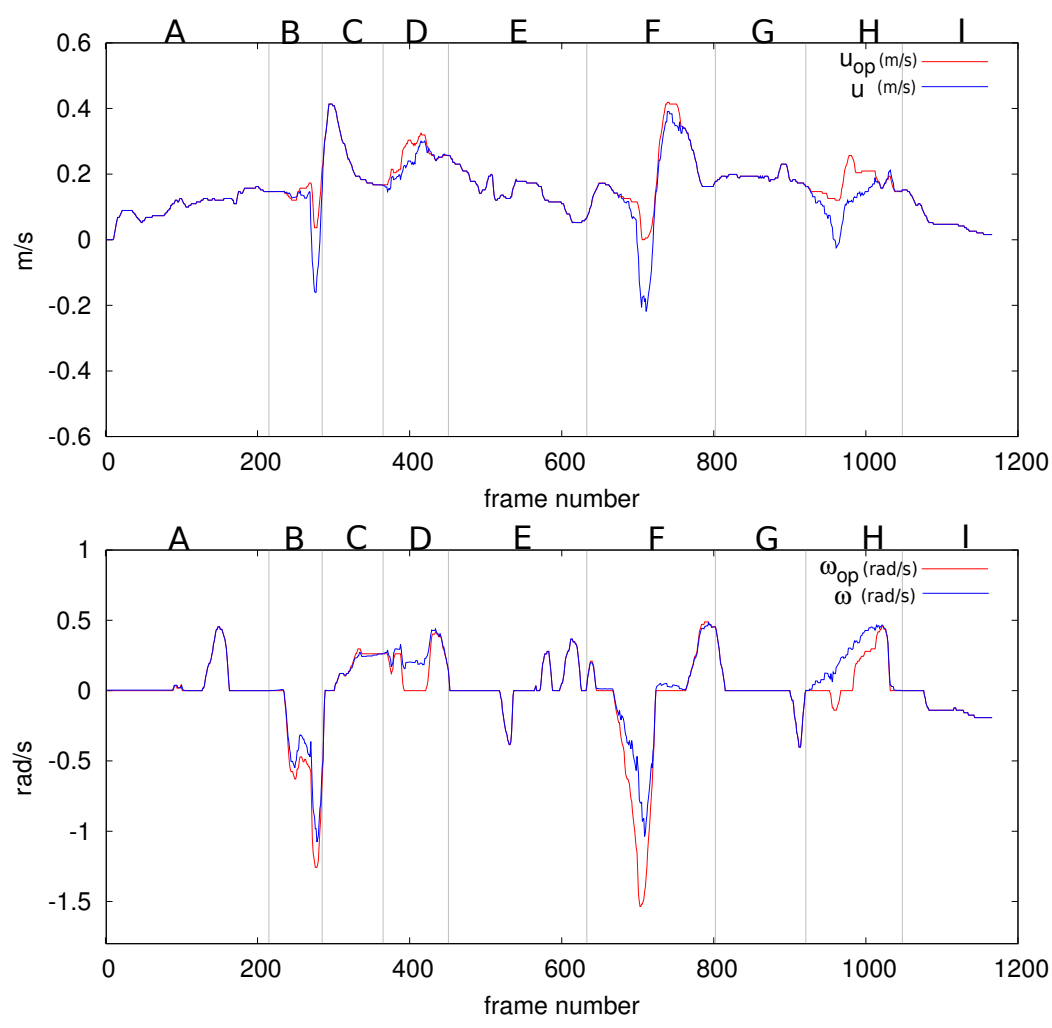

Figure 20: Evolution of $u$ along with $u_{o p}$ and $\omega$ along with $\omega_{o p}$. 

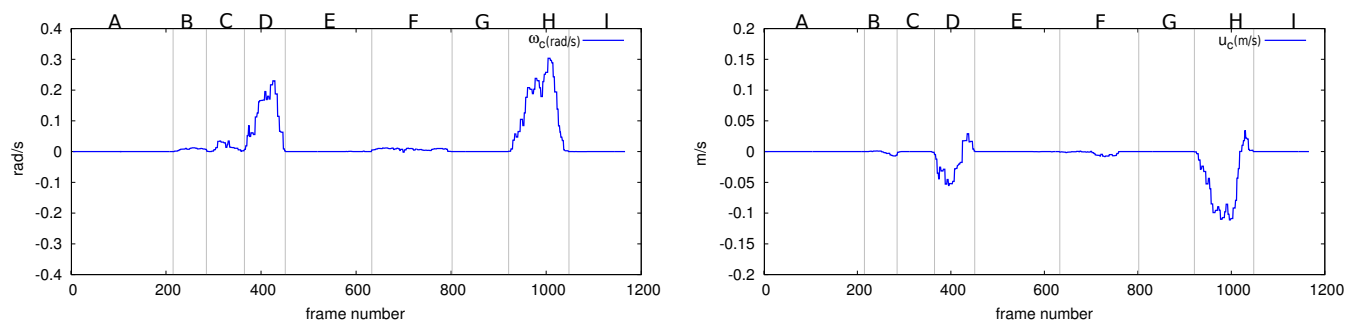

Figure 21: The corrective angular velocity $\left(\omega_{c}\right)$ and translational velocity $\left(u_{c}\right)$ for wall collision avoidance

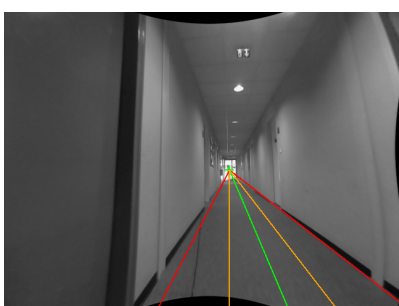

(a)

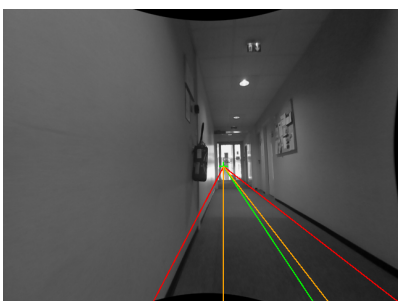

(b)

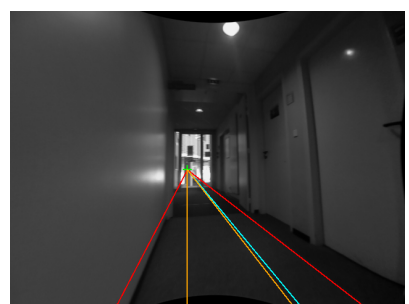

(c)

Figure 22: Camera frame snapshots pertaining to a corridor following experiment with force feedback where the user drove faster in a low illumination corridor.

from the orientation of the median line and the position of the vanishing point. As the activation factors $h_{x_{f}}$ and $h_{\theta_{m}}$ moves from zero to non-zero, it can be seen that the feedback force applied on the joystick increases (and decreases) in a near smooth manner. When considering the velocities, it can be observed that the user and the system velocities are not equal. But owing to the force feedback, the difference between the user and the system velocities is reduced. The corrective velocities in Figure 21 also show that the system does not have to automatically correct the trajectory even when there exists a risk of collision, especially during parts B,C and F. This is in contrast with the results presented in the above subsection where it can be seen that the difference between the user and the system velocities is proportional to the values of the activation factors $h_{x_{f}}$ and $h_{\theta_{m}}$. Moreover, the difference in user and system velocities can also be attributed to the force applied by the user against the feedback force provided by the system.

Thus, the feedback force serves as a corrective mechanism by itself which modifies the user teleoperation progressively and thus automatically corrects the motion of the wheelchair. Also, this force can be related as a guidance 


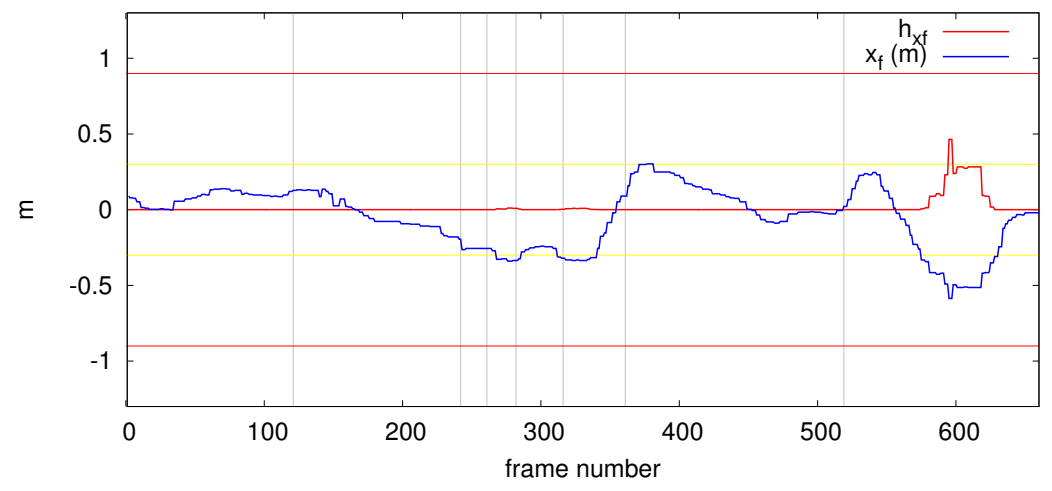

Figure 23: Visual feature $x_{f}$ along with activation factor $h_{x_{f}}$ (Faster Driving and Low Illumination).

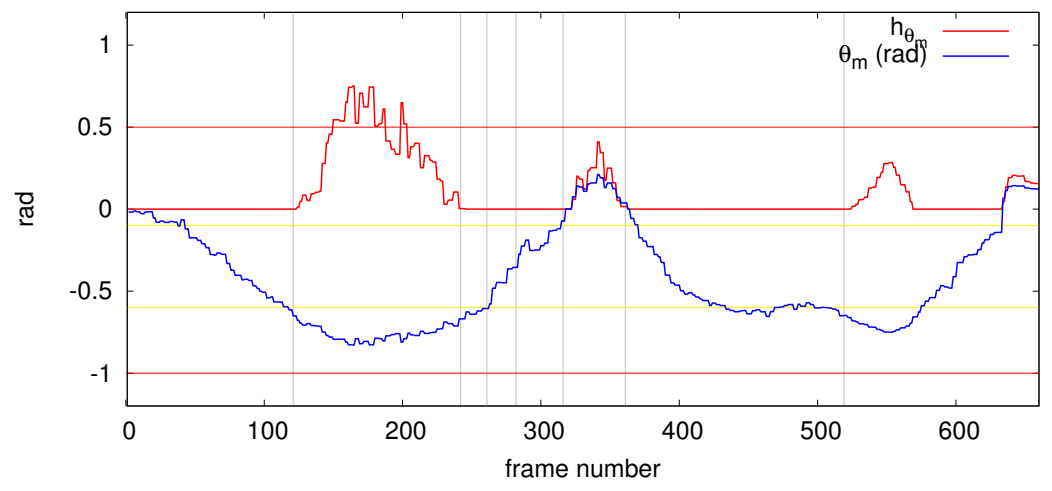

Figure 24: Visual feature $\theta_{m}$ along with activation factor $h_{\theta_{m}}$ (Faster Driving and Low Illumination).

tool which helps the user to identify the dangerousness of the situation and act accordingly. Therefore, it can be said that the proposed haptic feedback system serves as an effective tool for safe and intuitive trajectory correction.

Finally, part of another run of the same experiment is presented where wheelchair is driven at a much higher velocity than normal in a lowly lit corridor. Figure 25 displays the translational and rotational components of the user and the final system velocities. It can be seen that the average speed is much higher when compared to the two previous experiments. Moreover, Figure 22 shows the camera frames at selected instants where it can be seen that the illumination is darker but the evolution of the visual features (Fig- 

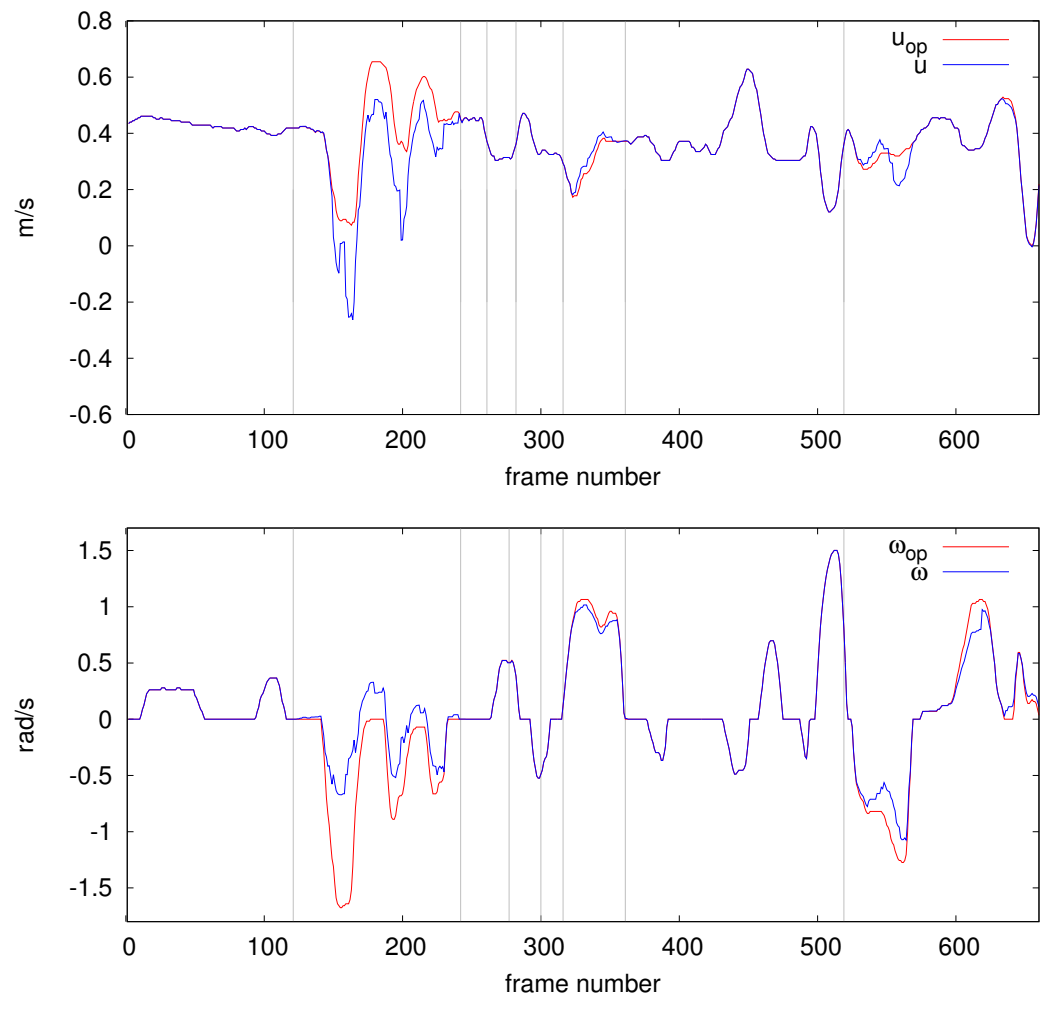

Figure 25: Evolution of $u$ along with $u_{o p}$ and $\omega$ along with $\omega_{o p}$ (Faster Driving and Low Illumination).

ures 23 and 24) remain consistent. This experiment verifies the robustness of the system particularly in cases where a wheelchair user may have erratic driving due to motor impairments.

The above results demonstrate the effectiveness of the proposed visionbased solution as a robust assistive system for the fundamental indoor navigation task of corridor following. A video of the results can be obtained from http://www.irisa.fr/lagadic/team/Marie.Babel-eng.html.

\section{Conclusion}

Preserving the individual autonomy and mobility is essential for the wellbeing of disabled people. In this work, we have proposed a vision-based semi-autonomous system designed for safe wheelchair navigation along cor- 
ridors. This control system relies on a collaboration between two tasks: first the manual steering and second wall avoidance task obtained by a dedicated visual servoing approach. The idea is then to correct the trajectory indicated by the user by servoing only the necessary degrees of freedom. This visual servoing process is based on both the vanishing point and wall plane detection. A smooth transition from manual driving to assisted navigation is obtained owing to an adapted weighting function, thus avoiding discontinuities that can lead to unpleasant experience. Results clearly show the ability of the approach to provide an efficient solution for wall avoiding purposes.

Moreover haptic force feedback in conjunction with the assistance was provided in order to notify the user of danger and guide him over to a safer zone. We observe that the guidance force serves as an automatic corrective mechanism which ensures minimal interference from the visual control process thus leading to a better quality of experience.

Future research aims at integrating local obstacle avoidance to the system while tackling other fundamental navigation problems like doorway passing. Delving into user intention analysis may also help in formulating adapted algorithms for vision-based wheelchair navigation assistance. A series of tests with the help of voluntary patients at a Rehabilitation Center (Pole St. Helier, Rennes, France) is also underway.

\section{Acknowledgement}

This work is supported by the Inria large-scale initiative action Personally Assisted Living (PAL). The authors would like to thank Daniel Guillard and Luc Le Pape from Ergovie, and Eric Bazin from INSA Rennes.

\section{References}

[1] B. Krieg-Brückner, D. Crombie, B. Gersdorf, A. Jüptner, M. Lawo, C. Mandel, A. B. Martínez, T. Röfer, C. Stahl, Challenges for Indoor and Outdoor Mobility Assistance, Technik für ein selbstbestimmtes Leben.

[2] A. Kokosy, T. Floquet, G. Howells, H. Hu, M. Pepper, C. Donzé, SYSIASS An Intelligent Powered Wheelchair, International Conference on Systems and Computer Science, 2012.

[3] L. Nordenfelt, Dignity in Care for Older People, Wiley-Blackwell, 2009.

[4] T. Gomi, A. Griffith, Developing intelligent wheelchairs for the handicapped, Assistive Technology and Artificial Intelligence, Vol. 1458 of 
Lecture Notes in Computer Science, Springer Berlin Heidelberg, 1998, pp. $150-178$.

[5] S. P. Levine, D. A. Bell, L. A. Jaros, R. C. Simpson, Y. Koren, J. Borenstein, The NavChair Assistive Wheelchair Navigation System, IEEE Transactions on Rehabilitation Engineering, 1999, pp. 443-451.

[6] E. Demeester, E. EB Vander Poorten, A. Hüntemann, J. De Schutter, Wheelchair navigation assistance in the FP7 project radhar: Objectives and current state, IROS Workshop on Progress, Challenges and Future Perspectives in Navigation and Manipulation Assistance for Robotic Wheelchairs, 2012.

[7] B. D. Argall, Modular and Adaptive Wheelchair Automation, Proceedings of the International Symposium on Experimental Robotics, Marrakech, Morrocco, 2014.

[8] R. A. M. Braga, M. Petry, A. P. Moreira, L. P. Reis, Intellwheels: A Development PlatForm for intelligent wheelchairs for disabled people, 5th International Conference on Informatics in Control, Automation and Robotics, 2008, pp. 115-121.

[9] F. Galán, M. Nuttin, E. Lew, P. W. Ferrez, G. Vanacker, J. Philips, J. D. R. Millán, A brain-actuated wheelchair: asynchronous and noninvasive Brain-computer interfaces for continuous control of robots., Clinical neurophysiology, 119 (9), 2008, pp. 2159-2169.

[10] T. Carlson, J. del R. Millan, Brain-Controlled Wheelchairs: A Robotic Architecture, IEEE Robotics \& Automation Magazine, 20 (1), 2013, pp. 65-73.

[11] S. Thrun, et al., Robotic mapping: A survey, Exploring artificial intelligence in the new millennium, 2002, pp. 1-35.

[12] N. Winters, J. Gaspar, G. Lacey, J. Santos-Victor, Omni-directional vision for robot navigation, Proceedings of IEEE Workshop on Omnidirectional Vision, 2000.

[13] R. Carelli, C. Soria, O. Nasisi, R. Freire, Stable agv corridor navigation with fused vision-based control signals, 28th Annual Conference of the Industrial Electronics Society, Vol. 3, 2002, pp. 2433-2438. 
[14] J. Toibero, C. Soria, F. Roberti, R. Carelliz, P. Fiorini, Switching visual servoing approach for stable corridor navigation, International Conference on Advanced Robotics, 2009, pp. 1-6.

[15] R. F. Vassallo, H. J. Schneebeli, J. Santos-Victor, Visual servoing and appearance for navigation, Robotics and Autonomous Systems, 31 (12), 2000, pp. $87-97$.

[16] R. C. Simpson, D. Poirot, F. Baxter, The hephaestus smart wheelchair system, IEEE Transactions on Neural Systems and Rehabilitation Engineering, 10 (2), 2002, pp. 118-122.

[17] J. Philips, J. del R. Millan, G. Vanacker, E. Lew, F. Galan, P. W. Ferrez, H. Van Brussel, M. Nuttin, Adaptive Shared Control of a BrainActuated Simulated Wheelchair, International Conference on Rehabilitation Robotics, 2007, pp. 408-414.

[18] R. Simpson, S. Levine, Adaptive shared control of a smart wheelchair operated by voice control, IEEE/RSJ International Conference on Intelligent Robot and Systems, Vol. 2, 1997, pp. 622-626.

[19] A. Escobedo, A. Spalanzani, C. Laugier, Using social cues to estimate possible destinations when driving a robotic wheelchair, IEEE/RSJ International Conference on Intelligent Robots and Systems, 2014, pp. 3299-3304.

[20] E. Vander Poorten, E. Demeester, E. Reekmans, J. Philips, A. Huntemann, J. De Schutter, Powered wheelchair navigation assistance through kinematically correct environmental haptic feedback, IEEE International Conference on Robotics and Automation, 2012, pp. 3706-3712.

[21] R. Luo, Force reflective feedback control for intelligent wheelchairs, IEEE/RSJ International Conference on Intelligent Robots and Systems, Vol. 2, 1999, pp. 918-923.

[22] L. Kitagawa, T. Kobayashi, T. Beppu, K. Terashima, Semi-autonomous obstacle avoidance of omnidirectional wheelchair by joystick impedance control, IEEE/RSJ International Conference on Intelligent Robots and Systems, Vol. 4, 2001, pp. 2148-2153. 
[23] A. Fattouh, M. Sahnoun, G. Bourhis, Force feedback joystick control of a powered wheelchair: preliminary study, IEEE International Conference on Systems, Man and Cybernetics, Vol. 3, 2004, pp. 2640-2645.

[24] G. Bourhis, M. Sahnoun, Assisted Control Mode for a Smart Wheelchair, International Conference on Rehabilitation Robotics, 2007, pp. 158-163.

[25] F. Chaumette, S. Hutchinson, Visual servo control, Part I: Basic approaches, IEEE Robotics and Automation Magazine, 13 (4), 2006, pp. 82-90.

[26] F. Pasteau, M. Babel, R. Sekkal, Corridor following wheelchair by visual servoing, IEEE/RSJ International Conference on Intelligent Robots and Systems, 2013, pp. 590-595.

[27] F. Pasteau, V. Karakkat-Narayanan, M. Babel, F. Chaumette, A visual servoing approach for autonomous corridor following and doorway passing in a wheelchair, Robotics and Autonomous Systems, 2015.

[28] F. Pasteau, A. Krupa, M. Babel, Vision-based assistance for wheelchair navigation along corridors, IEEE International Conference on Robotics and Automation, 2014.

[29] C. Rother, A new approach for vanishing point detection in architectural environments, 11th British Machine Vision Conference, 2000, pp. 382391.

[30] K. Boulanger, K. Bouatouch, S. Pattanaik, ATIP: A Tool for 3D Navigation inside a Single Image with Automatic Camera Calibration, EG UK conference, 2006.

[31] R. Grompone von Gioi, J. Jakubowicz,J.-M. Morel, G. Randall, LSD: a Line Segment Detector, Image Processing On Line, 2012.

[32] R. Sekkal, F. Pasteau, M. Babel, B. Brun, I. Leplumey, Simple Monocular door detection and tracking, IEEE International Conference on Image Processing, 2013.

[33] K. Ok, D.-N. Ta, F. Dellaert, Vistas and wall-floor intersection features - enabling autonomous flight in man-made environments, Workshop on Visual Control of Mobile Robots, 2012. 
[34] E. Delage, H. Lee, A. Ng, A dynamic bayesian network model for autonomous 3d reconstruction from a single indoor image, IEEE Conference on Computer Vision and Pattern Recognition, Vol. 2, 2006, pp. $2418-2428$.

[35] O. Kermorgant, F. Chaumette, Combining IBVS and PBVS to ensure the visibility constraint, IEEE/RSJ International Conference on Intelligent Robots and Systems, 2011, pp. 2849-2854.

[36] T. Li, O. Kermorgant, A. Krupa, Maintaining visibility constraints during tele-echography with ultrasound visual servoing, IEEE International Confernence on Robotics and Automation, 2012, pp. 4856-4861.

[37] N. Mansard, F. Chaumette, Task Sequencing for High-Level SensorBased Control, IEEE Transactions on Robotics, 23 (1), 2007, pp. 60-72.

[38] N. Mansard, A. Remazeilles, F. Chaumette, Continuity of VaryingFeature-Set Control Laws, IEEE Transactions on Automatic Control, 54 (11), 2009, pp. 2493-2505.

[39] C. Samson, B. Espiau, M. L. Borgne, Robot Control: The Task Function Approach, Oxfored University Press, 1991.

[40] M. Quigley, K. Conley, B. Gerkey, J. Faust, T. Foote, J. Leibs, R. Wheeler, A. Y. Ng, Ros: an open-source robot operating system, ICRA workshop on open source software, 3 (3.2), 2009.

[41] E. Marchand, F. Spindler, F. Chaumette, ViSP for visual servoing: a generic software platform with a wide class of robot control skills, IEEE Robotics and Automation Magazine, 12 (4), 2005, pp. 40-52. 